\title{
On Non-Cooperativeness in Social Distance Games
}

\author{
Alkida Balliu \\ Department of Computer Science, Aalto University, Finland \& \\ Gran Sasso Science Institute, Italy \\ Michele Flammini \\ Gran Sasso Science Institute \& DISIM - University of L'Aquila, Italy \\ Giovanna Melideo \\ DISIM - University of L'Aquila, Italy \\ Dennis Olivetti \\ Department of Computer Science, Aalto University, Finland \& \\ Gran Sasso Science Institute, Italy
}

ALKIDA.BALLIU@AALTO.FI

MICHELE.FLAMMINI@GSSI.IT

GIOVANNA.MELIDEO@UNIVAQ.IT

DENNIS.OLIVETTI@AALTO.FI

\begin{abstract}
We consider Social Distance Games (SDGs), that is cluster formation games in which the utility of each agent only depends on the composition of the cluster she belongs to, proportionally to her harmonic centrality, i.e., to the average inverse distance from the other agents in the cluster. Under a non-cooperative perspective, we adopt Nash stable outcomes, in which no agent can improve her utility by unilaterally changing her coalition, as the target solution concept. Although a Nash equilibrium for a SDG can always be computed in polynomial time, we obtain a negative result concerning the game convergence and we prove that computing a Nash equilibrium that maximizes the social welfare is NP-hard by a polynomial time reduction from the NP-complete Restricted Exact Cover by 3-Sets problem. We then focus on the performance of Nash equilibria and provide matching upper bound and lower bounds on the price of anarchy of $\Theta(n)$, where $n$ is the number of nodes of the underlying graph. Moreover, we show that there exists a class of SDGs having a lower bound on the price of stability of $\frac{6}{5}-\epsilon$, for any $\epsilon>0$. Finally, we characterize the price of stability of SDGs for graphs with girth 4 and girth at least 5 , the girth being the length of the shortest cycle in the graph.
\end{abstract}

\section{Introduction}

Coalition formation is a pervasive aspect of social life and it has been studied extensively in algorithmic game theory using the natural model of Hedonic Games (HGs), introduced by Dreze and Greenberg (1980) and further explored in several papers, such as Banerjee, Konishi, and Sönmez (2001); Bogomolnaia and Jackson (2002); and Cechlárová and Romero-Medina (2001). An HG consists of a set of selfish agents (humans, robots, software agents, etc.) having preferences over coalitions that might include them, regardless of which other coalitions may or may not be present. In other words, the utility of each agent only depends on the composition of the coalition she belongs to, without any form of externality, that is, without caring about the structure of the other coalitions. The outcome of an HG is a partition of the agents' set into disjoint coalitions (or clusters), referred to as a clustering or coalition structure.

Stability is the main criterion that has been used to analyze which coalition structures will arise: an outcome should be resistant to individual/group deviations, with different types of deviations giving rise to different notions of stability (such as core stability, individual stability, Nash stability). 
A significant stream of research (Aziz, Brandt, \& Harrenstein, 2013; Aziz, Brandt, \& Seedig, 2013; Bogomolnaia \& Jackson, 2002; Banerjee et al., 2001; Elkind \& Wooldridge, 2009; Gairing \& Savani, 2010) characterizes the existence and the properties of stable clusterings in hedonic games. For an overview, see the recent survey by Aziz and Savani (2016).

Under a non-cooperative perspective, we focus on Nash stable outcomes in which, unlike the core, every agent cannot coordinate with the others in order to understand if she can improve her utility. This notion of stability is better suited in settings in which it is not possible to assume the ability of coordination, or the cost of coordination is excessively high, like in huge distributed environments characterized by decentralization, autonomy, and general lack of cooperation among the network entities. A clustering is Nash stable, or it is a Nash equilibrium, if no agent can improve her utility by unilaterally changing her own coalition. In this setting, the outcome generated by such uncoordinated selfish agents can be inferior to a centrally designed one. Hence, one of the main issues is evaluating the induced degradation of the system performance. In particular, the most popular measure of the inefficiency of equilibria is the price of anarchy (PoA) (Koutsoupias \& Papadimitriou, 1999; Papadimitriou, 2001), defined as the worst-case ratio between the social welfare (i.e., the sum of the agents utilities) of a best clustering and the social welfare of a Nash stable clustering. Notice that for this definition to make sense, a game should allow at least one Nash equilibrium. Roughly speaking, a low PoA means that agents' selfish behaviour has a small impact on the system performance; a high PoA, instead, gives evidence that agents can really hurt the system and some sort of coordination is useful. Since a game has an high PoA even if only one of its multiple equilibria is highly inefficient, to differentiate between games in which all equilibria are inefficient and those in which only some equilibria are inefficient, we adopt a related optimistic measure, called the price of stability (PoS) (Anshelevich, Dasgupta, Kleinberg, Tardos, Wexler, \& Roughgarden, 2004), that is the best-case ratio between the social welfare of a best clustering and the social welfare of a Nash stable clustering. In other words, the PoS is related to the best possible outcome that selfish agents might accept. The PoS thus quantifies the necessary quality degradation caused by imposing the game-theoretic constraint of stability.

Since the number of possible coalitions and coalition structures is exponential, the mere specification of the input with all the corresponding values is already intractable. Therefore, researchers have focused on suitable subclasses allowing concise descriptions. Along this line, a growing interest in HGs has concerned instances in which agents entertain preferences over the other agents, which are then naturally lifted to preferences over coalitions. Additively Separable HGs (Banerjee et al., 2001) are a corresponding natural class of HGs, that can be succinctly represented by means of a preference graph of the agents. In these games, each agent has a value for any other one, and her utility for being in a coalition is simply the sum of the values she ascribes to its members, or analogously her degree centrality in the coalition. A related class of HGs is given by the Fractional Hedonic Games (FHGs) (Aziz, Brandt, \& Harrenstein, 2014), in which the utility of an agent is still given by her degree centrality in the own coalition, but it is also divided by the size of her cluster. Given that the utility of an agent is not simply defined as her total happiness, but as its ratio with the coalition size, FHGs can model several natural behavioral dynamics in social environments, like the well-known Bakers and Millers game.

In this paper we focus on Social Distance Games (SDGs), another important subclass of HGs introduced by Brânzei and Larson (2011), still defined around the notion of node centrality. In particular, they are based on the concept of social distance (i.e., the number of hops required to reach one node from another), which has become famous since Milgram's study on six degrees of 
separation. In SDGs the utility of an agent is given by the average inverse distance from all the other nodes in her coalition, that is by her harmonic centrality, divided by the size of the coalition. The basic idea is that each agent prefers to maintain stronger ties with closer ones, in such a way that also non neighbor agents contribute to her utility in a decreasing way with respect to their distance. The utility formulation is a variant of the closeness centrality and reflects the principle of homophily, that similarity breeds connection and people tend to form communities with similar others (McPherson, Lovin, \& Cook, 2001). Namely, it is related to several other classical measures from network analysis, such as degree, closeness, betweenness, and eigenvector centrality (Gomez, Gonzlez-Arangena, Manuel, Owen, del Pozo, \& Tejada, 2003), all of which are used to determine how a node is embedded in the network. Among such notions, the harmonic centrality used in SDGs has been identified as one of the best indexes, as it is the unique one satisfying a set of desirable properties (Boldi \& Vigna, 2014).

SDGs not only have the merit of being able to depart from the previous models that consider only the direct social neighborhood, but also provide a suitable abstraction of the utility definition, able to isolate the notion of node centrality in a modular way. Thus, they are capturing recent research attention and will possibly lead to other interesting models related to different suitable measures of social centrality.

\subsection{Related Work}

In the literature, an extensive research considered HGs from a strategic cooperative point of view, with the aim of characterizing the existence and the properties of coalition structures such as, for instance, the core (Bogomolnaia \& Jackson, 2002; Banerjee et al., 2001; Elkind \& Wooldridge, 2009; Gairing \& Savani, 2010). A clustering is core stable if there is no group of agents who can all be better off by forming a new deviating coalition. Non-cooperative studies on HGs in which selforganized clusterings are obtained from the decisions taken by independent and selfish agents can be found in Bloch and Diamantoudi (2011); Feldman, Lewin-Eytan, and Naor (2015); and Gairing and Savani (2010). More precisely, Banerjee et al. (2001) study properties guaranteeing the existence of core allocations for HGs with additively separable utilities, while Bogomolnaia and Jackson (2002) consider several forms of clustering stability like the core and the Nash. Aziz, Brandt, and Seedig (2011); Ballester (2004); and Olsen (2009) deal with computational complexity issues related to HGs, also considering additively separable utilities. In particular, Olsen (2009) shows that the problem of deciding whether a Nash stable clustering exists in an additively separable HG is NPcomplete, as well as the one of deciding whether a Nash stable clustering different from the grand coalition exists in an additively separable HG with non-negative and symmetric preferences. Bloch and Diamantoudi (2011) study non-cooperative games of coalition formation and identify conditions for stable outcomes. In a similar way, Apt and Witzel (2009) study how certain proposed rules can transform clusterings into other ones with specific properties. Feldman et al. (2015) investigate some interesting subclasses of HGs from a non-cooperative point of view, by characterizing Nash equilibria and providing upper and lower bounds on both the price of stability and the price of anarchy.

Fractional Hedonic Games are the class of games most related to the Social Distance Games considered in this paper. As already mentioned, they have been traditionally investigated under the additively-separable property. Namely, the utility of an agent is given by the sum of her preferences for each single member of her coalition, i.e. by her degree centrality, divided by the size of the 
coalition. FHGs have been investigated in Aziz et al. (2014); Aziz, Gaspers, Gudmundsson, Mestre, and Täubig (2015); and Brandl, Brandt, and Strobel (2015) from a cooperative perspective and in Bilò, Fanelli, Flammini, Monaco, and Moscardelli (2014); Bilò, Fanelli, Flammini, Monaco, and Moscardelli (2015); and Olsen (2012) from a non-cooperative viewpoint. In particular, we point out that Aziz et al. (2014) focus on core stable partitions and present a number of classes of graphs for which the core is non-empty, including graphs with girth at least 5 , the girth being the length of the shortest cycle in the graph.

Olsen (2012) introduces a modified version of FHGs further investigated in Kaklamanis, Kanellopoulos, and Papaioannou (2016); and Monaco, Moscardelli, and Velaj (2018), where the utility of an agent is divided by the size of the coalition she belongs to minus 1. Authors study the existence, complexity and performance of solutions that lead to Nash, strong Nash, and core stable outcomes.

Apart from examining standard stability notions, Aziz et al. (2013); and Elkind, Fanelli, and Flammini (2016) investigate Pareto optimality in HGs and FHGs. Pareto optimal solutions have been in fact considered in HGs as outcomes arising from the strategic interaction of the agents, as they are stable under the deviation of the grand coalition. In fact, they do not permit a simultaneous deviation by all the agents making all of them weakly better off and some strictly better off. In such papers the authors give intractability results on the determination of Pareto optimal solutions and suitable bounds on their price of anarchy, called price of Pareto optimality in this setting.

Flammini, Monaco, Moscardelli, Shalom, and Zaks (2018) study HGs where agents come in an online fashion, one at a time. They consider additively separable HGs and FHGs and show tight bounds in the cases where the edge-weights can be constant, positive, or unrestricted.

Flammini, Monaco, and Zhang (2017) study strategyproof mechanisms for additively separable HGs and FHGs. More precisely, they show partitioning methods without payments such that agents do not gain anything, utility wise, by lying about their true preferences.

To the best of our knowledge Social Distance Games have been considered in the cooperative context of core stability by Brânzei and Larson (2011). As already observed, they are related to FHGs in the sense that, in the definition of agents utilities, the degree centrality measure is substituted by the harmonic centrality. Brânzei and Larson (2011) claim that finding the best clustering can be shown to be NP-hard on SDGs via a reduction from the NP-complete Partition into Triangles problem, and provide an algorithm to approximate the optimal welfare within a factor of 2 . They analyze core stable solutions, determining their welfare and stability characteristics, and showing that they possess the small world property.

Balliu, Flammini, and Olivetti (2017b) investigate Pareto stability in SDGs and show that, while computing a Pareto stable solution maximizing the social welfare in bounded degree graphs is NPhard, a suitably approximating one can be determined in polynomial time. They also provide asymptotically tight bounds on the price of Pareto optimality for several classes of social graphs.

\subsection{Our Contribution}

In this paper we approach SDGs from the viewpoint of non-cooperative game theory with the aim of understanding the existence, computability and performances of Nash stable clusterings. We first focus on the existence of Nash stable clusterings: even if SDGs always admit a Nash equilibrium, we show that they may not converge to Nash equilibria and then give a polynomial time reduction from the NP-complete RESTRICTED EXACT COVER by 3-SETS $(R X C 3)$ problem (Gonzalez, 1985) to prove that it is NP-hard to find a best Nash equilibrium. We then study the performances of the Nash 
equilibria and provide matching upper and lower bounds on the price of anarchy of $\Theta(n)$, where $n$ is the number of nodes of the underlying graph. Also, we show that there exists a class of SDGs having a lower bound on the price of stability of $\frac{6}{5}-\epsilon$, for any arbitrarily small $\epsilon>0$. Finally, we characterize the price of stability of SDGs for graphs with girth 4 and girth at least 5 , the girth being the length of the shortest cycle in the graph. Our results are summarized in Tables 1 and 2.

Notice that graphs with limited girth, while generalizing known topologies such as bipartite graphs and being among the classical ones investigated in this setting, seem to identify the SDGs for which good stable solutions can be computed in polynomial time. In fact, for a girth greater than 4 we prove that, by partitioning the nodes into stars, it is possible to efficiently determine a stable solution which has a social welfare at least equal to the optimal one divided by 1.207 . On the other hand, as we will show in the sequel, there are graphs with girth at most 4 that do not admit a stable star partition.

We finally remark that, after the preliminary version of this paper appeared in Balliu, Flammini, Melideo, and Olivetti (2017a), an improved lower bound of 2 of the price of stability for SDGs on general graphs has been provided by Kaklamanis, Kanellopoulos, and Patouchas (2018). In that paper, the authors have also proven that the price of stability in trees is 1 ; moreover, they have studied the price of stability for a modified version of SDGs, where the utility is computed with respect to the cluster size minus 1.

\begin{tabular}{l|l} 
Existence of a Nash equilibrium & Always \\
\hline Convergence to a Nash equilibrium & Not guaranteed \\
\hline Finding a best Nash equilibrium & NP-hard
\end{tabular}

Table 1: Nash equilibria in SDGs.

\begin{tabular}{c|c|c|c} 
& Lower Bound & Upper Bound & Graph Topology \\
\hline \multirow{3}{*}{$\operatorname{PoS}$} & $6 / 5-\epsilon$ & $?$ & general \\
\cline { 2 - 4 } & $169 / 160$ & $?$ & girth 4 \\
\cline { 2 - 4 } & $?$ & $1 / 2+1 / \sqrt{2}$ & girth greater that 4 \\
\hline PoA & $\Omega(n)$ & $O(n)$ & general
\end{tabular}

Table 2: Price of Stability and Price of Anarchy in SDGs.

\section{Model and Preliminaries}

Consider an undirected graph $G=(V, E)$. A coalition or cluster is a non-empty subset of $V$. The set of all nodes $V$ is called the grand coalition, whereas a coalition of size 1 is said a singleton coalition and its node singleton or isolated. For any integer $n>0$ denote with $[n]$ the set of integers 
$\{1, \ldots, n\}$ and with $[k, n]$, for $k \leq n$ the subset $\{k, \ldots, n\} \subseteq[n]$. A clustering or coalition structure is a partition of $V$ into $k>0$ coalitions $\mathcal{C}=\left\{C_{1}, \ldots, C_{k}\right\}$ such that $C_{i} \subseteq V$ for each $i \in[k], \bigcup_{i \in[k]} C_{i}=V$ and $C_{i} \cap C_{j}=\emptyset$ for any $i, j \in[k]$ with $i \neq j$. Given a coalition $C \subseteq V$, we denote by $G(C)$ the subgraph induced by $C$. For brevity, we will often identify $G(C)$ directly with the corresponding coalition $C$ and we say that a coalition $C$ is connected or disconnected if $G(C)$ is so.

Let the harmonic centrality of a node $x \in V$ in an undirected graph $G=(V, E)$ be defined as $\mu_{x}(G)=\sum_{y \in V \backslash\{x\}} \frac{1}{d_{G}(x, y)}$, where for any pair of nodes $x, y \in V, d_{G}(x, y)$ denotes the length of a shortest path connecting $x$ and $y$ in $G$. The sum of the inverse of the social distances can be viewed as the similarity of a node in its graph, and it indicates its centrality.

We consider coalition forming games in which each node $x \in V$ is an agent. More precisely, we focus on the following class of games.

Definition 2.1 (Brânzei \& Larson, 2011) A Social Distance Game $S D G(G)$, or SDG in short, is represented as an undirected graph $G=(V, E)$ where ( $i) V$ is the set of $n$ agents and (ii) the utility of an agent $x \in V$ in a given coalition $C$ is a suitable function of her harmonic centrality in the subgraph induced by $C$ and of the size of $C$, that is:

$$
u_{x}(C)=\frac{\mu_{x}(G(C))}{|C|}= \begin{cases}0 & \text { if } C=\{x\} \\ \frac{1}{|C|} \sum_{y \in C \backslash\{x\} \frac{1}{d_{C}(x, y)}} & \text { otherwise }\end{cases}
$$

If $x$ and $y$ are disconnected in $C$, then $d_{C}(x, y)=\infty$.

We denote by $\mathcal{C}(x)$ the coalition of $\mathcal{C}$ including node $x$. Let us use $u_{x}(\mathcal{C})$ as a shorthand for the utility $u_{x}(\mathcal{C}(x))$ of $x$ in a given clustering $\mathcal{C}$. Each agent chooses the coalition to belong to with the aim of maximizing her utility. A solution or outcome of a SDG is a clustering $\mathcal{C}$. The social welfare $S W(\mathcal{C})$ of a clustering $\mathcal{C}$ is the sum of the agents' utilities, i.e., $S W(\mathcal{C})=\sum_{x \in V} u_{x}(\mathcal{C})$.

In the rest of this paper, we can assume that the input graph is non-singleton and connected, since disconnected graphs can be analyzed componentwise.

For any pair of agents $x$ and $y$, we denote with $(\mathcal{C}, x, y)$ the clustering obtained from $\mathcal{C}$ by moving $x$ from $\mathcal{C}(x)$ to $\mathcal{C}(y)$. A clustering $\mathcal{C}^{\prime}=(\mathcal{C}, x, y)$ is an improving deviation for the agent $x$ in $\mathcal{C}$ if $u_{x}(\mathcal{C})<u_{x}\left(\mathcal{C}^{\prime}\right)$. In this case we say that agent $x$ makes an improving move. An improvement path is a sequence of improving deviations. A game has the finite improvement property (FIP) if every improvement path of the game is finite.

An agent $x$ is Nash stable in a clustering $\mathcal{C}$ if she cannot perform any improving move, that is if $\forall y \in V, u_{x}(\mathcal{C}) \geq u_{x}\left(\mathcal{C}^{\prime}\right)$, where $\mathcal{C}^{\prime}=(\mathcal{C}, x, y)$. A clustering $\mathcal{C}$ is Nash stable, or a Nash equilibrium, if every agent $x$ is Nash stable in $\mathcal{C}$. If an improvement path is finite then its last clustering is a Nash equilibrium.

We are interested in bounding the performances of Nash equilibria with respect to the social optimum OpT, i.e. the social welfare of a best clustering $\mathcal{C}^{*}$ that maximizes $S W\left(\mathcal{C}^{*}\right)$, that is such that $\mathrm{OPT}=S W\left(\mathcal{C}^{*}\right)=\max _{\mathcal{C}} S W(\mathcal{C})$. Notice that $\mathcal{C}^{*}$ is not necessarily an equilibrium. If $\mathcal{N}$ denotes the set of the Nash equilibria, a best (worst) Nash equilibrium is a Nash equilibrium $\mathcal{C} \in \mathcal{N}$ that maximizes (minimizes) $S W(\mathcal{C})$. The best (worst) social cost is the social welfare of a best (worst) Nash equilibrium. The price of anarchy (PoA) is the ratio between the social optimum and the worst social cost in a game i.e., $\mathrm{PoA}=\max _{\mathcal{C} \in \mathcal{N}} \frac{\mathrm{OPT}}{S W(\mathcal{C})}$; the price of stability $(\mathrm{PoS})$ is the ratio between the social optimum and the best social cost, i.e., $\operatorname{PoS}=\min _{\mathcal{C} \in \mathcal{N}} \frac{\mathrm{OPT}}{\operatorname{SW}(\mathcal{C})}$. 


\section{Nash Equilibria: Existence, Convergence and Complexity of their Finding}

In order to establish the existence of Nash equilibria for SDGs and to prove that determining a Nash equilibrium can be done in polynomial time, it is sufficient to notice that the grand coalition is Nash stable. In fact, no agent can have any improving move by deciding to form a singleton coalition on her own. Thus, on the basis of these considerations, we can state the following:

Proposition 1 A SDG always admits a Nash equilibrium and it can be computed in polynomial time.

\subsection{Convergence to Equilibrium in SDGs}

In the sequel of this section we investigate if, starting from an arbitrary initial clustering, an improvement path is always guaranteed to converge to a Nash equilibrium, and we provide a negative answer. In fact, Theorem 1 shows that SDGs may admit an infinite sequence of improving deviations (i.e., the FIP property is not guaranteed).

\section{Theorem 1 SDGs may not converge to Nash equilibria.}

Proof. Consider the instance $\operatorname{SDG}(G)$ where $G$ is the bipartite graph depicted in Figure 1 with vertex set $V=X \cup Y \cup Z$, where $X=\left\{x_{i} \mid i \in[20]\right\}, Y=\left\{y_{i} \mid i \in[15]\right\}$ and $Z=\left\{z_{1}, z_{2}\right\}$. In the following, for $i \leq j$, we write $X_{i, j}$ and $Y_{i, j}$ as a shorthand for $\left\{x_{i}, \ldots, x_{j}\right\} \subseteq X$ and $\left\{y_{i}, \ldots, y_{j}\right\} \subseteq Y$, respectively.

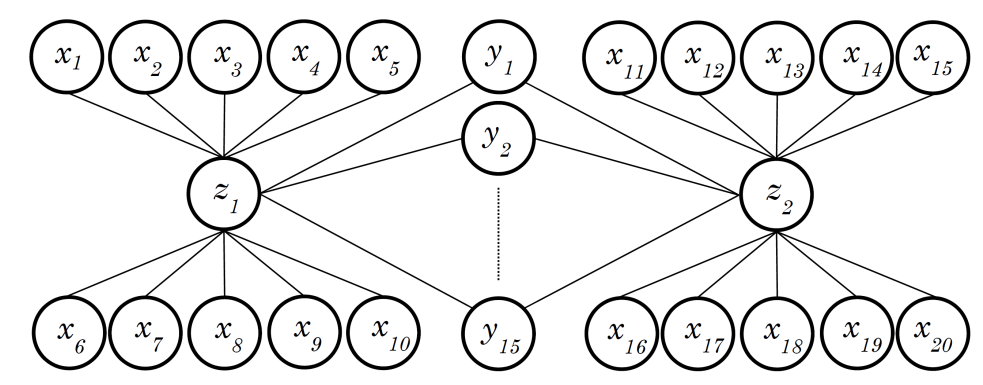

Figure 1: A SDG not converging to a Nash equilibrium.

Starting from the clustering $\mathcal{C}=\left\{X_{1,10} \cup\left\{z_{1}\right\}, Y, X_{11,20} \cup\left\{z_{2}\right\}\right\}$ depicted in Figure 2, we prove the existence of a cyclic sequence of improving deviations which shows the claim.

Namely, we consider the following improvement path:

- $\mathcal{C}^{(1)}=\left(\mathcal{C}, z_{1}, y_{1}\right)=\left\{X_{1,10}, Y \cup\left\{z_{1}\right\}, X_{11,20} \cup\left\{z_{2}\right\}\right\}$ (see Figure 3 ) is the improving deviation obtained from $\mathcal{C}$ by moving $z_{1}$ to $\mathcal{C}\left(y_{1}\right)$. Since $u_{z_{1}}(\mathcal{C})=\frac{10}{11}$ and $u_{z_{1}}\left(\mathcal{C}^{(1)}\right)=\frac{15}{16}>\frac{10}{11}$, agent $z_{1}$ can really make an improving move.

- $\mathcal{C}^{(2)}=\left(\mathcal{C}^{(1)}, z_{2}, z_{1}\right)=\left\{X_{1,10}, Y \cup Z, X_{11,20}\right\}$ (see Figure 4) is the improving deviation resulting from $\mathcal{C}^{(1)}$ by moving agent $z_{2}$ in $\mathcal{C}^{(1)}\left(z_{1}\right)$. In fact $z_{2}$ can improve her utility from $u_{z_{2}}\left(\mathcal{C}^{(1)}\right)=\frac{10}{11}$ to $u_{z_{2}}\left(\mathcal{C}^{(2)}\right)=u_{z_{2}}(Y \cup Z)=\frac{15+\frac{1}{2}}{17}=\frac{31}{34}$ by moving to $\mathcal{C}^{(1)}\left(z_{1}\right)=Y \cup\left\{z_{1}\right\}$. 

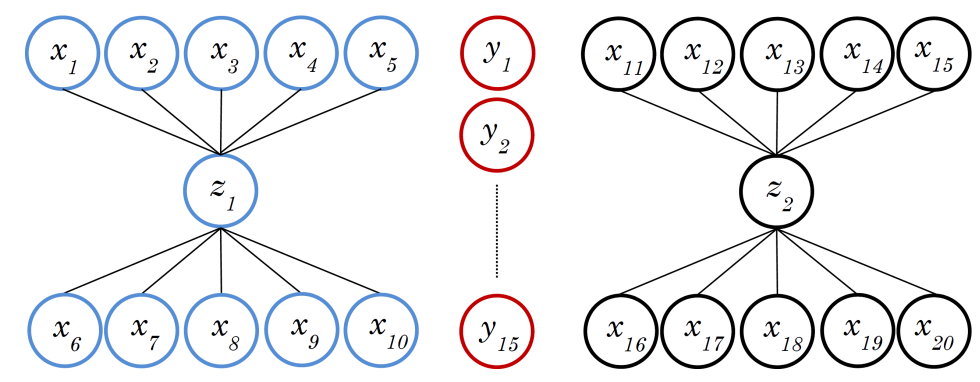

Figure 2: The initial clustering $\mathcal{C}$, where $u_{z_{1}}(\mathcal{C})=u_{z_{2}}(\mathcal{C})=\frac{10}{11}$.

- Since $\forall x \in X, u_{x}\left(\mathcal{C}^{(2)}(x)\right)=0$, agents $x_{1}, \ldots, x_{5}$ and $x_{11}, \ldots, x_{15}$ can increase their utility by moving one after another to the coalition including agents in $Y \cup Z$. Thus starting from $\mathcal{C}^{(2)}$, we consider 10 improving moves inducing the clustering $\mathcal{C}^{(12)}=\left\{X_{6,10}, Y \cup Z \cup X_{1,5} \cup\right.$ $\left.X_{11,15}, X_{16,20}\right\}$ depicted in Figure 5 .

- Notice that $u_{z_{1}}\left(\mathcal{C}^{(12)}\right)=\frac{20+\frac{1}{2}+\frac{5}{3}}{27}=\frac{133}{162}$ and thus agent $z_{1}$ can increase her utility to $\frac{5}{6}$ by moving back to $X_{6,10}$. Thus $\mathcal{C}^{(13)}=\left(\mathcal{C}^{(12)}, z_{1}, x_{6}\right)=\left\{X_{6,10} \cup\left\{z_{1}\right\}, Y \cup\left\{z_{2}\right\} \cup X_{1,5} \cup\right.$ $\left.X_{11,15}, X_{16,20}\right\}$ (see Figure 6).

- Since $u_{z_{2}}\left(\mathcal{C}^{(13)}\right)=\frac{10}{13}$, agent $z_{2}$ induces an improving deviation by moving back to $X_{16,20}$, that is $\mathcal{C}^{(14)}=\left(\mathcal{C}^{(13)}, z_{2}, x_{16}\right)=\left\{X_{6,10} \cup\left\{z_{1}\right\}, Y \cup X_{1,5} \cup X_{11,15}, X_{16,20} \cup\left\{z_{2}\right\}\right\}$ and $u_{z_{2}}\left(\mathcal{C}^{(14)}\right)=\frac{5}{6}>\frac{10}{13}$ (see Figure 7$)$.

- Agents $x_{1}, \ldots, x_{5}$ and $x_{11}, \ldots, x_{15}$ have now utility zero in $\mathcal{C}^{(14)}$ and thus they can increase the utility by moving back one after another to the cluster containing $z_{1}$ and $z_{2}$, respectively. Then $\mathcal{C}$ is again reached and therefore the claim follows.

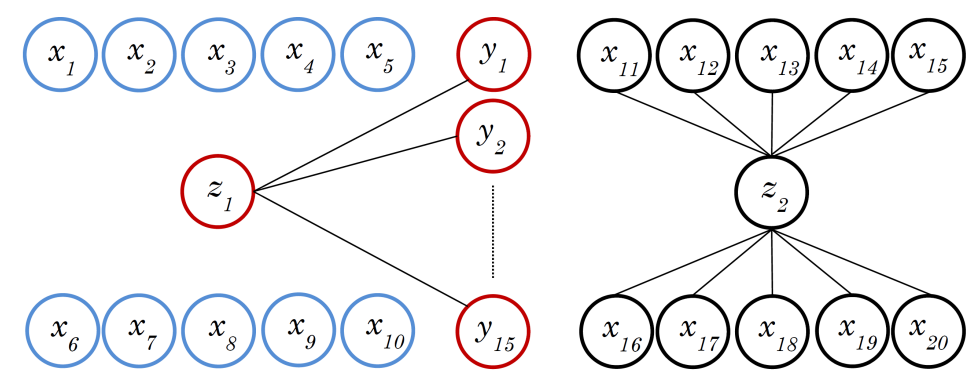

Figure 3: $\mathcal{C}^{(1)}=\left(\mathcal{C}, z_{1}, y_{1}\right)$, where $u_{z_{1}}\left(\mathcal{C}^{(1)}\right)=\frac{15}{16}>\frac{10}{11}$.

\subsection{NP-completeness of Finding a Best Nash Equilibrium for SDGs}

While determining the existence of a Nash equilibrium can be trivially done in polynomial time, as the grand coalition is stable, in the sequel of this section we show that unfortunately computing 


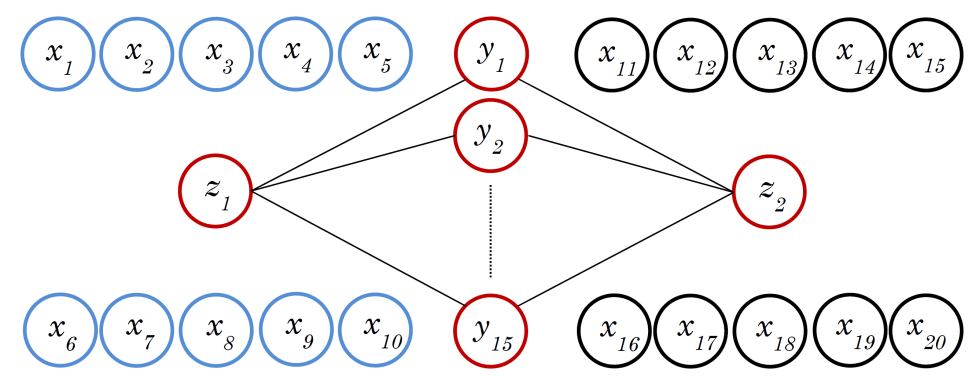

Figure 4: $\mathcal{C}^{(2)}=\left(\mathcal{C}^{(1)}, z_{2}, z_{1}\right)$, where $u_{z_{1}}\left(\mathcal{C}^{(2)}\right)=u_{z_{2}}\left(\mathcal{C}^{(2)}\right)=\frac{15+\frac{1}{2}}{17}=\frac{31}{34}>\frac{10}{11}$.

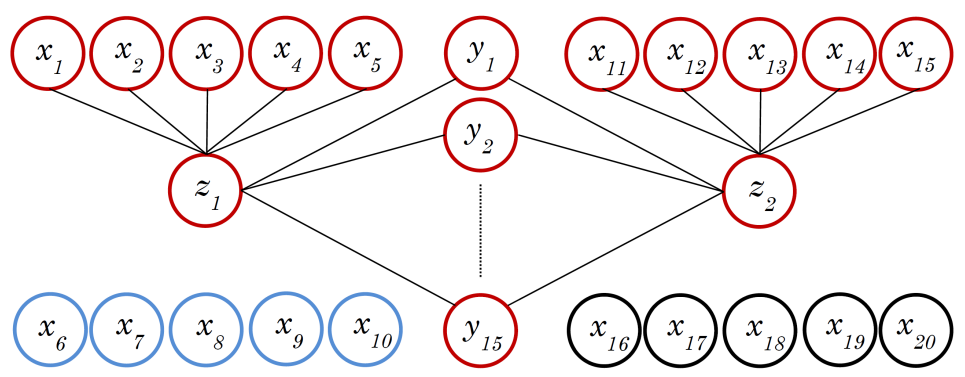

Figure 5: $\mathcal{C}^{(12)}=\left\{X_{6,10}, Y \cup Z \cup X_{1,5} \cup X_{11,15}, X_{16,20}\right\}$, where $u_{z_{1}}\left(\mathcal{C}^{(12)}\right)=\frac{133}{162}$.

a best Nash equilibrium for SDGs is NP-hard. To this aim, we provide a polynomial time reduction from the NP-complete RESTRICTED EXACT Cover by 3-SETS ( $R X C 3$ ) problem (Gonzalez, 1985) which is defined as follows.

Restricted Exact Cover by 3-Sets ( $R \boldsymbol{X} \boldsymbol{C} 3)$ problem. Let $m=3 p$ for some integer $p \geq 1$. An instance of the $R X C 3$ problem consists of a universe set $U=\left\{u_{1}, \ldots, u_{m}\right\}$ of $m$ elements and a collection $\mathcal{B}=\left\{B_{1}, \ldots, B_{m}\right\}$ of 3 -elements subsets of $U$ (triples) such that $\bigcup_{j=1}^{m} B_{j}=U$ and each element of $U$ appears exactly in three triples. The problem is to decide if $\mathcal{B}$ contains an exact cover for $U$, i.e. a subcollection $\mathcal{F} \subseteq \mathcal{B}$ such that every element of $U$ occurs in exactly one triple of $\mathcal{F}$.

In the following we show how to transform an instance of $R X C 3$ to a suitable instance of SDG with the technique of local replacement. Roughly speaking, we use a subgraph to represent every triple in $\mathcal{B}$ (see Figure 8) and we show that the composition of these subgraphs is a graph with certain properties that has a Nash stable clustering with social welfare at least $\frac{257}{20} p$ if and only if $\mathcal{B}$ contains an exact cover.

NP-hardness Reduction from $\boldsymbol{R} \boldsymbol{X} \boldsymbol{C} 3$ to SDG. Suppose we are given an instance $\mathcal{I}=(U, \mathcal{B})$ of $R X C 3$. We can define an instance of SDG by specifying the underlying undirected graph $G_{\mathcal{I}}=$ $(V, E)$ as follows:

- For each of the $m$ elements $u \in U$, we have a node $y \in V$. For convenience, for all $i \in$ $[m]$ we denote by $Y_{i}=\left\{y_{i, 1}, y_{i, 2}, y_{i, 3}\right\}$ the set of nodes associated with the triple $B_{i}=$ $\left(u_{i, 1}, u_{i, 2}, u_{i, 3}\right) \in \mathcal{B}$. Thus, let $Y=\cup_{i=1}^{m} Y_{i}$ be the set of the $m$ nodes associated with $U$. For each triple $B_{i} \in \mathcal{B}$, with $i \in[m]$, we consider a set of four nodes $K_{i}=\left\{z_{i, 1}, z_{i, 2}, z_{i, 3}, z_{i, 4}\right\}$ 


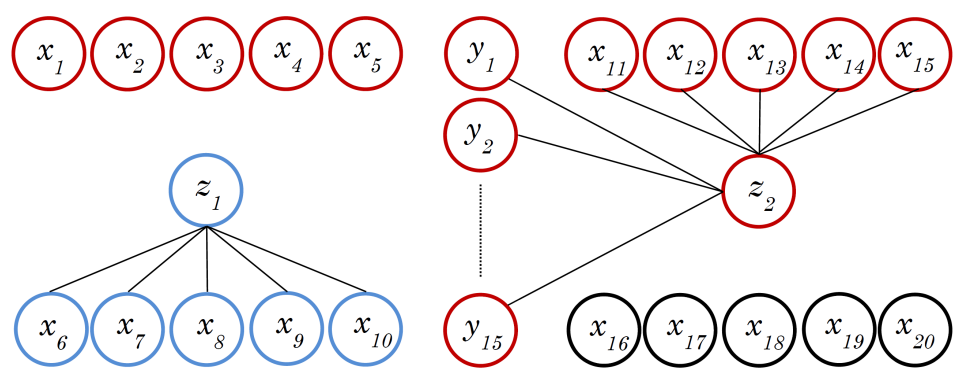

Figure 6: $\mathcal{C}^{(13)}=\left(\mathcal{C}^{(12)}, z_{1}, x_{6}\right)=\left\{X_{6,10} \cup\left\{z_{1}\right\}, Y \cup\left\{z_{2}\right\} \cup X_{1,5} \cup X_{11,15}, X_{16,20}\right\}$ where $u_{z_{1}}\left(\mathcal{C}^{(13)}\right)=\frac{5}{6}>\frac{133}{162}$.

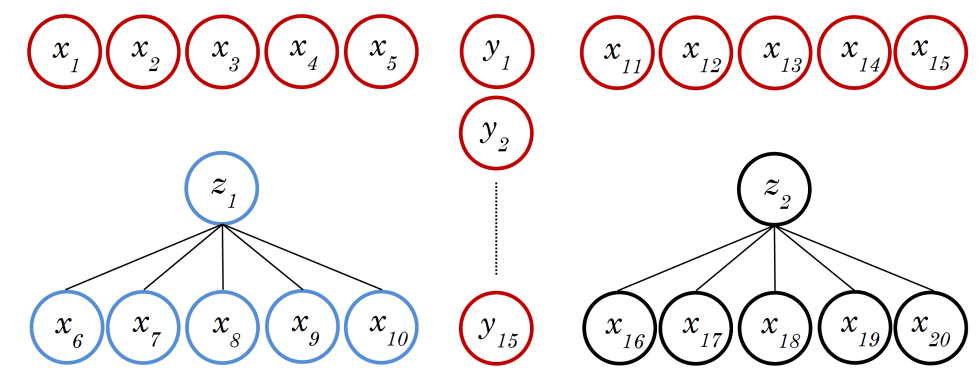

Figure 7: $\mathcal{C}^{(14)}=\left(\mathcal{C}^{(13)}, z_{2}, x_{16}\right)=\left\{X_{6,10} \cup\left\{z_{1}\right\}, Y \cup X_{1,5} \cup X_{11,15}, X_{16,20} \cup\left\{z_{2}\right\}\right\}$ where $u_{z_{2}}\left(\mathcal{C}^{(14)}\right)=\frac{5}{6}>\frac{10}{13}$.

and one additional node $x_{i}$. Therefore, if $X=\left\{x_{1}, \ldots, x_{m}\right\}$ and $K=\cup_{i=1}^{m} K_{i}$, we have that $V=K \cup X \cup Y$, with $|V|=6 m$.

- For what concerns the set of edges $E$, for each $i \in[m], G_{\mathcal{I}}$ contains one 4-clique $K_{4}\left(K_{i}\right)$ which includes all vertices in $K_{i}$ (i.e., six edges), a set of three edges $\left\{\left(x_{i}, z_{i, 2}\right),\left(x_{i}, z_{i, 3}\right)\right.$,$\left.\left(x_{i}, z_{i, 4}\right)\right\}$ and a set of further three edges $\left\{\left(x_{i}, y_{i, 1}\right),\left(x_{i}, y_{i, 2}\right),\left(x_{i}, y_{i, 3}\right)\right\}$. Consequently, it must be $|E|=12 \mathrm{~m}$.

As an example, a triple $B_{i}=\left(u_{i, 1}, u_{i, 2}, u_{i, 3}\right) \in \mathcal{B}$ of $R X C 3$ corresponds to the subgraph of $G_{\mathcal{I}}$ depicted in Figure 8. We notice that, since each element of $U$ appears exactly in three triples, each $y \in Y$ has exactly three incident edges.

Clearly such a reduction can be done in polynomial time. In order to complete the proof and prove the hardness, we show that the constructed SDG instance $G_{\mathcal{I}}$ has a Nash stable clustering with social welfare at least $\frac{257}{20} p$ if and only if the $R X C 3$ instance $\mathcal{I}$ has an exact cover.

Before proving the main theorem, let us analyze some fundamental properties of Nash stable clusterings for $G_{\mathcal{I}}$. 


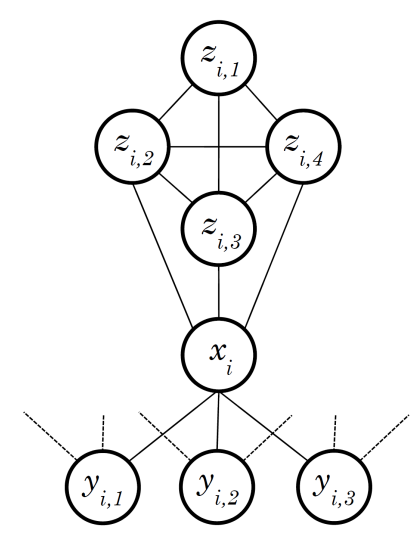

Figure 8: Subgraph of $G_{\mathcal{I}}$ associated with a triple $B_{i}=\left(u_{i, 1}, u_{i, 2}, u_{i, 3}\right) \in \mathcal{B}$ of $R X C 3$.

\subsubsection{STRUCTURAL PROPERTIES OF STABLE CLUSTERINGS FOR ANY INSTANCE $G_{\mathcal{I}}$ OF SDGs.}

In the following we discuss some structural properties of Nash stable clusterings for $G_{\mathcal{I}}$. In particular, Lemma 1 stresses that agents of $K_{i}$ (for $i \in[m]$ ) must always belong to the same coalition, whereas Lemmas 2 and 3 deal with the cases in which agents of $K_{i}$ must belong to the same coalition as $x_{i}$.

Lemma 1 Let $\mathcal{C}$ be any Nash stable clustering for an instance $G_{\mathcal{I}}$ of $S D G$. For all $i \in[m]$, agents in $K_{i}$ are always included in the same coalition.

Proof. For any $i \in[m]$, denote by $C_{1}=\mathcal{C}\left(z_{i, 1}\right)$ the coalition containing $z_{i, 1}$ in the stable clustering $\mathcal{C}$. We show that, in order to guarantee stability, it must be $K_{i} \subseteq C_{1}$. Arguing by contradiction, assume that there exists an agent in $K_{i}$ which is not included in $C_{1}$. Without loss of generality, let $z_{i, 4} \notin C_{1}$ such an agent and let $C_{4}=\mathcal{C}\left(z_{i, 4}\right)$.

A case analysis shows that such an assumption leads to a contradiction. We can distinguish different cases based on the cardinality of $C_{1}$ and $C_{4}$. Since in a stable clustering an agent cannot be isolated, $C_{1}$ and $C_{4}$ must contain at least an agent adjacent to $z_{i, 1}$ and $z_{i, 4}$, respectively, and therefore $\left|C_{1}\right| \geq 2$ and $\left|C_{4}\right| \geq 2$.

- First of all, assume that $\left|C_{1}\right|=2$, and let $C_{1}=\left\{z_{i, 1}, z_{i, 2}\right\}$. We can distinguish three cases depending on the cardinality of $C_{4}$ :

- $\left|C_{4}\right|=2$. If $C_{4}=\left\{x_{i}, z_{i, 4}\right\}, z_{i, 3}$ cannot be Nash stable as it is disconnected, i.e., $u_{z_{i, 3}}(\mathcal{C})=0$, and therefore she can improve her utility by moving to $C_{1}$ or $C_{4}$. If $C_{4}=\left\{z_{i, 3}, z_{i, 4}\right\}$, all agents in $K_{i}$ are not Nash stable, as each of them can make an improving move to the other coalition and achieve a utility of $\frac{2}{3}>\frac{1}{2}$. Both cases lead to a contradiction. Clearly, $z_{i, 4}$ would be disconnected and not stable in any other coalition.

- $\left|C_{4}\right|=3$. The only coalition without disconnected agents is $C_{4}=\left\{z_{i, 3}, z_{i, 4}, x_{i}\right\}$. Nevertheless, agents $z_{i, 1}$ and $z_{i, 2}$, with $u_{z_{i, 1}}\left(C_{1}\right)=u_{z_{i, 2}}\left(C_{1}\right)=\frac{1}{2}$, can improve by moving to $C_{4}$, as they would achieve utilities $u_{z_{i, 1}}\left(\left(C_{1}, z_{i, 1}, z_{i, 4}\right)\right)=\frac{2+\frac{1}{2}}{4}=\frac{5}{8}$ and $u_{z_{i, 2}}\left(\left(C_{1}, z_{i, 2}, z_{i, 4}\right)\right)=\frac{3}{4}$ : a contradiction. 
- $\left|C_{4}\right|>3$. In such a case it must necessarily be $\left\{z_{i, 3}, z_{i, 4}, x_{i}\right\} \subset C_{4}$. Since $u_{z_{i, 4}}=$ $u_{z_{i, 3}} \leq \frac{2+\frac{\left|C_{4}\right|-3}{2}}{\left|C_{4}\right|}=\frac{\left|C_{4}\right|+1}{2\left|C_{4}\right|}=\frac{1}{2}+\frac{1}{2\left|C_{4}\right|}<\frac{1}{2}+\frac{1}{6}=\frac{2}{3}$, agents $z_{i, 3}$ and $z_{i, 4}$ can make an improving move to $C_{1}$ achieving a utility of $\frac{2}{3}$ : a contradiction.

- Assume now that $\left|C_{1}\right|=3$. If $C_{1}=\left\{z_{i, 1}, z_{i, 2}, z_{i, 3}\right\}$, any possible coalition $C_{4}$ without

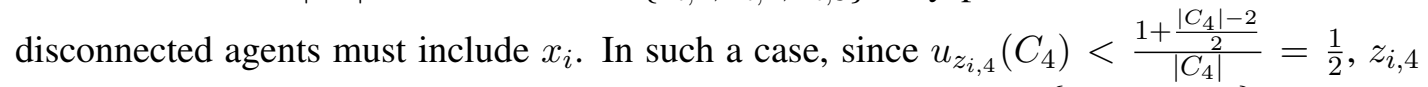
can improve her utility by moving to $C_{1}$. On the other hand, if $C_{1}=\left\{z_{i, 1}, z_{i, 2}, x_{i}\right\}$, it must be $C_{4}=\left\{z_{i, 3}, z_{i, 4}\right\}$. Agents $z_{i, 3}$ and $z_{i, 4}$ can move to $C_{1}$, whereas $z_{i, 1}$ and $x_{i}$ can move to $C_{4}$, improving their utilities from $\frac{1}{2}$ to $\frac{3}{4}$ and $\frac{2}{3}$, respectively. Both cases lead to a contradiction.

- Finally, in order to conclude the proof, let us consider the case $\left|C_{1}\right| \geq 4$. Since by assumption $C_{1} \neq K_{i}$, we have that $x_{i} \in C_{1}$. Thus, it must be $\left\{z_{i, 1}, z_{i, 2}, x_{i}\right\} \subseteq C_{1}$ and $C_{4}=\left\{z_{i, 3}, z_{i, 4}\right\}$. Since $u_{z_{i, 1}}\left(C_{1}\right) \leq \frac{1+\frac{1}{2}+\frac{\left|C_{1}\right|-3}{3}}{\left|C_{1}\right|}=\frac{6+3+2\left|C_{1}\right|-6}{6\left|C_{1}\right|}=\frac{3+2\left|C_{1}\right|}{6\left|C_{1}\right|}=\frac{1}{2\left|C_{1}\right|}+\frac{1}{3}<\frac{1}{6}+\frac{1}{3}=\frac{1}{2}$, agent $z_{i, 1}$ can make an improving move to $C_{4}$, by achieving a utility of $\frac{2}{3}$ : a contradiction.

Lemma 1 guarantees that in any Nash stable clustering for an instance $G_{\mathcal{I}}$ of SDG, all the four agents in $K_{i}$ are always included in the same coalition, for all $i \in[m]$. However, a 4-clique $K_{i}$ may be together with some other $K_{j}$ in a coalition $C$. The remaining structural properties, aiming at characterizing conditions under which a stable coalition can include one or more $K_{i}$, depend on how many agents $x_{i} \in X$ are included in a same connected coalition. Namely, Lemmas 2 and 3 distinguish the two cases where a Nash stable coalition $C$ contains one or more agents of $X$. We will refer to the coalitions containing at most one agent $x_{i} \in X$ as basic coalitions or (basic) $x_{i}$ coalitions to emphasize that they just contain $x_{i}$. On the other hand, coalitions including at least two agents of $X$ are called composed coalitions.

Lemma 2 Let $\mathcal{C}$ be any Nash stable clustering for an instance $G_{\mathcal{I}}$ of $S D G$. For any basic $x_{i}$ coalition $C \in \mathcal{C}$ containing at most two agents of $Y_{i}$, agents in $K_{i}$ must belong to $C$ as well, i.e., $K_{i} \subset C$. On the other hand, if $C$ contains exactly three agents of $Y_{i}$, then $K_{i}$ may not be included in $C$.

Proof. Let $C$ be a Nash stable basic $x_{i}$-coalition in $\mathcal{C}$ including at most two agents of $Y_{i}$ (just like coalitions $t_{0}, t_{1}, t_{2}$ depicted in Figure 9). By Lemma 1, since in $\mathcal{C}$ all the agents in $K_{i}$ are within the same coalition, the cases are two: either $K_{i} \subseteq C$ (as we want to prove) or $K_{i} \not \subset C$. Assume by contradiction that $K_{i} \not \subset C$. In such a case $|C| \leq 3$, and thus $u_{x_{i}}(C) \leq \frac{2}{3}$. Therefore, agent $x_{i}$ could improve her utility by moving to $K_{i}$, as she would achieve utility $u_{x_{i}}\left(K_{i} \cup\left\{x_{i}\right\}\right)=\frac{7}{10}>\frac{2}{3}$ : a contradiction. Thus, in order to guarantee stability, it must be $K_{i} \subset C$.

On the other hand, we can notice that if $C=\left\{x_{i}, y_{i, 1}, y_{i, 2}, y_{i, 3}\right\}$, coalition $K_{i}$ is actually stable even if $K_{i} \not \subset C$ (see the two coalitions corresponding to type $t_{4}$ in Figure 9). In fact, no agent in $K_{i}$ can make an improving move by moving to $C$, because agent $z_{i, 1}$ would obtain a null utility, whereas agents $z_{i, 2}, z_{i, 3}, z_{i, 4}$ would decrease their utility from $\frac{3}{4}$ to $\frac{1}{2}$. Moreover, by moving to any other coalition in $\mathcal{C}$, all agents in $K_{i}$ would be disconnected and would obtain a utility zero. We can also stress that, by moving from $C$ to $K_{i}$, agent $x_{i}$ would decrease her utility from $\frac{3}{4}$ to $\frac{7}{10}$, and agents $y_{i, 1}, y_{i, 2}, y_{i, 3}$ would get utility zero. 
It is worthwhile underlining that by Lemmas 1 and 2, Nash stable basic coalitions can take only the forms depicted in Figure 9, where the subfigure $t_{4}$ actually shows a pair of coalitions, i.e., $K_{i}$ and $Y_{i} \cup\left\{x_{i}\right\}$.

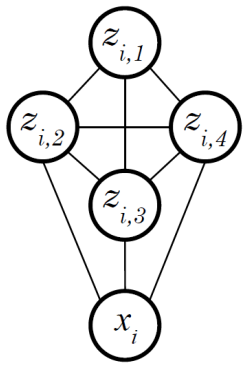

$\left(\mathrm{t}_{0}\right)$

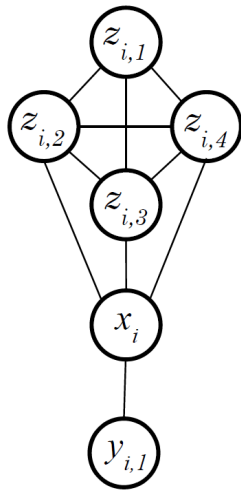

$\left(\mathrm{t}_{1}\right)$

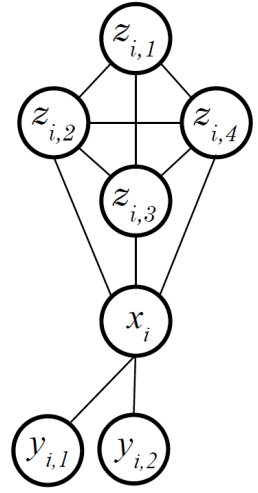

$\left(\mathrm{t}_{2}\right)$

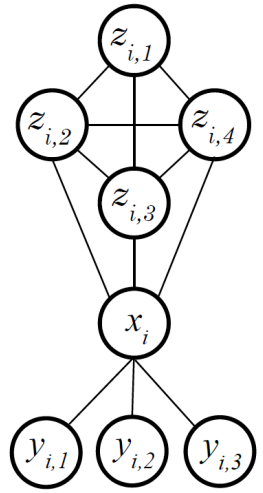

$\left(t_{3}\right)$

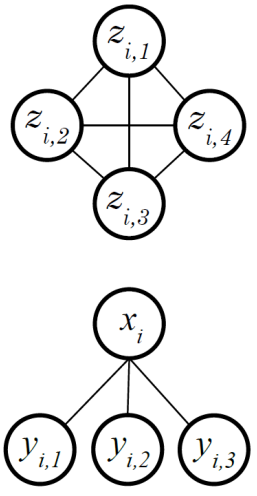

$\left(\mathrm{t}_{4}\right)$

Figure 9: Nash stable basic coalitions for any instance $G_{\mathcal{I}}$ of SDG.

The following structural result holds for composed coalitions.

Lemma 3 Let $\mathcal{C}$ be any Nash stable clustering for an instance $G_{\mathcal{I}}$ of $S D G$. If two agents $x_{i}$ and $x_{j}$ of $X$ belong to a same connected composed coalition $C=\mathcal{C}\left(x_{i}\right)=\mathcal{C}\left(x_{j}\right)$, then agents in $K_{i}$ and $K_{j}$ must belong to the same coalition as well, i.e., $K_{i} \cup K_{j} \subset C$.

Proof. First of all we notice that if $x_{i}$ and $x_{j}$ belong to the same Nash stable connected coalition $C$, there must exist a path between them. Thus, there exists at least a node $y \in Y_{i} \cap Y_{j}$ such that $\left\{x_{i}, x_{j}, y\right\} \subseteq C$ (see Figure 10).

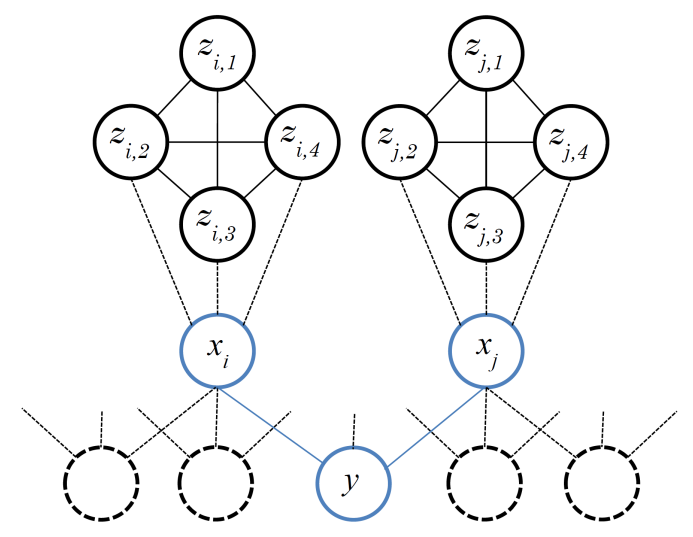

Figure 10: A stable connected composed coalition containing $x_{i}$ and $x_{j}$. 
It is worth recalling that by Lemma 1 all the four agents in $K_{i}$ (resp. $K_{j}$ ) must be together in a same coalition. Arguing by contradiction, assume that $K_{i} \not \subset C$. A case analysis shows that, in order to guarantee stability, agents in $K_{i}$ must be in $C$.

Let $\nu_{i}=\left|Y_{i} \cap C\right| \geq 1$ and $\nu_{j}=\left|Y_{j} \cap C\right| \geq 1$ denote the number of agents adjacent to $x_{i}$ and $x_{j}$ which are in $Y_{i}$ and $Y_{j}$, respectively. We stress that, if $K_{i} \not \subset C$, agent $x_{i}$ has $\nu_{i} \leq 3$ agents at distance $1,1+\alpha$ agents at distance 2 (included $x_{j}$ ), for $\alpha \geq 0$, and all the other $\beta \geq 0$ agents at distance at least 3. Namely:

$$
u_{x_{i}}(C) \leq \frac{\nu_{i}+\frac{1+\alpha}{2}+\frac{\beta}{3}}{\nu_{i}+2+\alpha+\beta}=\frac{6 \nu_{i}+3+3 \alpha+2 \beta}{6\left(\nu_{i}+2+\alpha+\beta\right)} .
$$

Assume first that $\nu_{i} \leq \nu_{j}$. We notice that when $\nu_{i}=\nu_{j}=3$ there must be at least one agent in $Y_{j} \cap C$ adjacent to $x_{j}$ at distance 3 , and then $\beta \geq 1$. Thus, Equation (1) implies that:

$$
u_{x_{i}}(C) \leq \begin{cases}\frac{9+3 \alpha+2 \beta}{6(3+\alpha+\beta)} \leq \frac{3(3+\alpha+\beta)}{6(3+\alpha+\beta)}=\frac{1}{2} & \text { if } \nu_{i}=1 \\ \frac{15+3 \alpha+2 \beta}{6(4+\alpha+\beta)}<\frac{4(4+\alpha+\beta)}{6(4+\alpha+\beta)}=\frac{2}{3} & \text { if } \nu_{i}=2 \\ \frac{21+3 \alpha+2 \beta}{6(5+\alpha+\beta)} \leq \frac{4(5+\alpha+\beta)+1}{6(5+\alpha+\beta)}=\frac{2}{3}+\frac{1}{6(5+\alpha+\beta)} \leq \frac{2}{3}+\frac{1}{36}=\frac{25}{36}<\frac{7}{10} & \text { if } \nu_{i}=3\end{cases}
$$

All these cases lead to a contradiction, since agent $x_{i}$ could always improve her utility by moving from $C$ to $K_{i}$. In fact, $u_{x_{i}}\left(K_{i} \cup\left\{x_{i}\right\}\right)=\frac{3+\frac{1}{2}}{5}=\frac{7}{10}>u_{x_{i}}(C)$ (see Equation (2)) and therefore, if $\nu_{i} \leq \nu_{j}$, agents in $K_{i}$ must be included in $C$.

Assume now that $\nu_{i}>\nu_{j}$. By applying the previous arguments to $x_{j}$, agents in $K_{j}$ must be in $C$. If by contradiction $K_{i} \not \subset C$ but $K_{j} \subset C$, agent $x_{i}$ has $\nu_{i} \leq 3$ agents at distance 1 (notice that $\nu_{i} \geq 2$ since $\nu_{j} \geq 1$ ), $1+\alpha$ agents at distance 2 (included $x_{j}$ ), for $\alpha \geq 0,4$ agents at distance at least $3\left(z_{i, 1}, z_{i, 2}, z_{i, 3}, z_{i, 4}\right)$ and further $\beta \geq 0$ agents at distance at least 3 . That is:

$$
\begin{aligned}
u_{x_{i}}(C) & \leq \frac{\nu_{i}+\frac{1+\alpha}{2}+\frac{4+\beta}{3}}{\nu_{i}+6+\alpha+\beta}=\frac{6 \nu_{i}+11+3 \alpha+2 \beta}{6\left(\nu_{i}+6+\alpha+\beta\right)}<\frac{3\left(\nu_{i}+6+\alpha+\beta\right)+3 \nu_{i}}{6\left(\nu_{i}+6+\alpha+\beta\right)} \\
& =\frac{1}{2}+\frac{\nu_{i}}{2\left(\nu_{i}+6+\alpha+\beta\right)} \leq \frac{1}{2}+\frac{3}{2(2+6)}=\frac{1}{2}+\frac{3}{16}=\frac{11}{16}<\frac{7}{10}
\end{aligned}
$$

which is a contradiction since $x_{i}$ could improve her utility to $\frac{7}{10}$ by moving from $C$ to $K_{i}$. Therefore, if $\nu_{i}>\nu_{j}$, agents in $K_{i}$ must be in $C$ as well.

The claim follows by applying symmetric arguments to $x_{j}$.

\subsubsection{STABILITY PRESERVING TRANSFORMATIONS OF INSTANCES $G_{\mathcal{I}}$ OF SDGS.}

In the following we provide some transformation rules for coalitions in Nash stable clusterings, which obtain a strictly higher social welfare. Indeed, we first prove that every composed coalition in a stable clustering for $G_{\mathcal{I}}$ can be split in basic coalitions leading to a new clustering with a strictly higher social welfare (Proposition 4). Then, in Lemma 4, we prove that starting from such a (not necessarily stable) clustering, the game tends to converge to a stable clustering with at least the same social welfare, still composed only of basic coalitions. Splitting every resulting coalition of type $t_{3}$ in two subcoalitions corresponding to type $t_{4}$ preserves stability and further increases the 
social welfare (Corollary 1). Therefore, we can finally state that a best Nash equilibrium for an instance $G_{\mathcal{I}}$ of SDG can always be found among clusterings including only basic coalitions of type $t_{0}, t_{1}, t_{2}, t_{4}$ (Corollary 2 ).

Let us preliminarly compute a lower bound on the average utility of the agents in any basic coalition of type $t_{0}, t_{1}, t_{2}, t_{4}$ (Proposition 2) and an upper bound on the agents' utilities in any stable composed coalition (Proposition 3 ).

Proposition 2 In any basic coalition of type $t_{0}, t_{1}, t_{2}, t_{4}$ the average utility of the agents in the coalition is at least 0.639 .

Proof. With reference to the coalitions depicted in Figure 9, we can compute the social welfare as follows:

$$
\begin{aligned}
S W\left(t_{0}\right) & =\frac{3 \cdot 4+2\left(3+\frac{1}{2}\right)}{5}=\frac{19}{5}=3.8 ; \\
S W\left(t_{1}\right) & =\frac{\left(3+\frac{1}{2}+\frac{1}{3}\right)+4\left(4+\frac{1}{2}\right)+\left(1+\frac{3}{2}+\frac{1}{3}\right)}{6}=\frac{\frac{23}{6}+18+\frac{17}{6}}{6}=\frac{37}{9} \approx 4.1 ; \\
S W\left(t_{2}\right) & =\frac{\left(3+\frac{1}{2}+\frac{2}{3}\right)+3 \cdot 5+\left(5+\frac{1}{2}\right)+2\left(1+\frac{4}{2}+\frac{1}{3}\right)}{7}=\frac{\frac{25}{6}+15+\frac{11}{2}+\frac{20}{3}}{7} \\
& =\frac{94}{21} \approx 4.47 ; \\
S W\left(t_{4}\right) & =\left(4 \cdot \frac{3}{4}\right)+\left(\frac{3}{4}+3 \cdot \frac{1+\frac{2}{2}}{4}\right)=3+\frac{9}{4}=\frac{21}{4}=5.25 .
\end{aligned}
$$

The claims follows by noting that by Equations (3-6), the average utility $\bar{u}$ of agents in basic coalitions of type $t_{0}, t_{1}, t_{2}, t_{4}$ is: $\bar{u}\left(t_{0}\right)=\frac{\frac{19}{5}}{5}=\frac{19}{25}=0.76, \bar{u}\left(t_{1}\right)=\frac{\frac{37}{9}}{6}=\frac{37}{54}>0.68, \bar{u}\left(t_{2}\right)=$ $\frac{\frac{94}{21}}{7}=\frac{94}{147}>0.639, \bar{u}\left(t_{4}\right)=\frac{\frac{21}{4}}{8}=\frac{21}{32}>0.656$.

Proposition 3 Let $\mathcal{C}$ be any Nash stable clustering for an instance $G_{\mathcal{I}}$ of SDG. In any connected composed coalition the utility of all the agents in the coalition is strictly less than 0.635 .

Proof. Let $C=\mathcal{C}\left(x_{i}\right)=\mathcal{C}\left(x_{j}\right)$ be a connected composed coalition including agents $x_{i}, x_{j} \in X$. By Lemma 3, we know that $K_{i} \cup K_{j} \subseteq C$ and that there must be a path between $x_{i}$ and $x_{j}$. Thus, we can give an upper bound to the utility of all agents in $C$ as follows (see Figure 10).

- Each $z_{i, 1}$ has at least 3 agents at distance $1\left(z_{i, 2}, z_{i, 3}, z_{i, 4}\right)$, one agent at distance $2\left(x_{i}\right)$, one agent at distance $4\left(x_{j}\right), 3$ agents at distance $5\left(z_{j, 2}, z_{j, 3}, z_{j, 4}\right)$, and one agent at distance 6 $\left(z_{j, 1}\right)$. All the other $\alpha$ agents (including the ones in $Y_{i} \cup Y_{j}$ ) have distance at least 3 , so that $u_{z_{i, 1}}(C) \leq \frac{3+\frac{1}{2}+\frac{\alpha}{3}+\frac{1}{4}+\frac{3}{5}+\frac{1}{6}}{10+\alpha}<0.46$.

- Each $z_{i, 2}$ (and symmetrically $z_{i, 3}$ and $z_{i, 4}$ ) has at least 4 agents at distance $1\left(z_{i, 1}, z_{i, 3}, z_{i, 4}, x_{i}\right)$, one agent at distance $3\left(x_{j}\right), 3$ agents at distance $4\left(z_{j, 2}, z_{j, 3}, z_{j, 4}\right)$, and one agent at distance $5\left(z_{j, 1}\right)$. All the other $\alpha$ agents have distance at least 2 , and thus $u_{z_{i, 2}}(C) \leq \frac{4+\frac{\alpha}{2}+\frac{1}{3}+\frac{3}{4}+\frac{1}{5}}{10+\alpha} \leq$ $\frac{317}{600}<0.53$. 
- Each $x_{i}$ can have at most 6 agents at distance $1\left(z_{i, 2}, z_{i, 3}, z_{i, 4}, y_{i_{1}}, y_{i_{2}}, y_{i_{3}}\right), 2$ agents at distance $2\left(z_{i, 1}, x_{j}\right), 3$ agents at distance $3\left(z_{j, 2}, z_{j, 3}, z_{j, 4}\right)$, and one agent at distance $4\left(z_{j, 1}\right)$. All the other $\alpha$ nodes have distance at least 2 , so that $u_{x_{i}}(C) \leq \frac{6+\frac{\alpha+2}{2}+\frac{3}{3}+\frac{1}{4}}{13+\alpha} \leq \frac{33}{52}<0.635$.

- Each $y \in Y \cap C$ has at most 3 agents at distance $1\left(x_{i}, x_{j}, x_{k}\right), 6$ agents at distance 2 $\left(z_{i, 2}, z_{i, 3}, z_{i, 4}, z_{j, 2}, z_{j, 3}, z_{j, 4}\right)$, and 2 agents at distance $3\left(z_{i, 1}, z_{j, 1}\right)$. All the other $\alpha$ nodes have distance at least 2 . This gives an upper bound to the utility of $y$ of $\frac{3+\frac{6+\alpha}{2}+\frac{2}{3}}{12+\alpha} \leq \frac{5}{9}<0.56$.

Based on the above results, Proposition 4 analyzes how the social welfare of a stable clustering $\mathcal{C}$ changes when partitioning every disconnected coalition into connected subcoalitions, and then splitting every resulting connected composed coalition in basic coalitions of type $t_{0}, t_{1}, t_{2}, t_{4}$.

Proposition 4 Let $\mathcal{C}$ be any Nash stable clustering for an instance $G_{\mathcal{I}}$ of $S D G$. All the composed coalitions $C \in \mathcal{C}$ can be split in basic coalitions of type $t_{0}, t_{1}, t_{2}, t_{4}$, obtaining a new (non necessarily stable) clustering $\overline{\mathcal{C}}$ with a strictly higher social welfare.

Proof. Consider any clustering $\overline{\mathcal{C}}$ obtained from $\mathcal{C}$ by first partitioning every disconnected coalition $C \in \mathcal{C}$ into connected subcoalitions, and then later splitting every connected composed coalition in subcoalitions of type $t_{0}, t_{1}, t_{2}, t_{4}$.

First of all, let $C \in \mathcal{C}$ be a disconnected coalition and let $k>1$ denote the number of connected subcoalitions $C_{1}, \ldots, C_{k}$ in $C$, such that $C_{1} \cap \ldots \cap C_{k}=\emptyset$ and $C=C_{1} \cup \ldots \cup C_{k}$. Moreover, let $\mathcal{C}^{\prime}$ be the clustering obtained from $\mathcal{C}$ by partitioning $C$ into the $k$ connected subcoalitions $C_{1}, \ldots, C_{k}$, i.e., $\mathcal{C}^{\prime}=\mathcal{C} \cup\left\{C_{1}, \ldots, C_{k}\right\} \backslash\{C\}$.

Clearly, $u_{x}\left(\mathcal{C}^{\prime}\right)=u_{x}(\mathcal{C})$ for all $x \in V \backslash C$. On the other hand, since $\mu_{x}(G(C))=\mu_{x}\left(G\left(C_{i}\right)\right)$ for every $x \in C_{i}$, it must be $u_{x}\left(\mathcal{C}^{\prime}\right)=\frac{\mu_{x}\left(G\left(C_{i}\right)\right)}{\left|C_{i}\right|}>\frac{\mu_{x}(G(C))}{|C|}=u_{x}(\mathcal{C}), \forall x \in C$. This proves that the clustering obtained from $\mathcal{C}$ by partitioning every disconnected coalition $C$ into connected subcoalitions has a strictly higher social welfare.

Now, let $\overline{\mathcal{C}}$ be any clustering obtained by further splitting every resulting connected composed coalition in basic coalitions of type $t_{0}, t_{1}, t_{2}, t_{4}$. Therefore, since the worst average utility 0.639 of the agents in any basic coalition of type $t_{0}, t_{1}, t_{2}, t_{4}$ (see Proposition 2 ) is greater than the best utility 0.635 of all the agents in the composed coalitions (see Proposition 3), the social welfare actually strictly increases in any $\overline{\mathcal{C}}$ as well. This proves the claim.

Notice that all the coalitions of type $t_{3}$ possibly contained within the Nash stable clustering $\mathcal{C}$ remain unchanged in $\overline{\mathcal{C}}$ after the abovementioned transformations. Therefore, such clustering $\overline{\mathcal{C}}$ can actually include only coalitions of the five types $t_{0}, \ldots, t_{4}$ depicted in Figure 9.

In the following, with a little abuse of language, sometimes we will refer to coalitions of type $t_{i}$ directly as coalitions $t_{i}$, for $i \in\{0, \ldots, 4\}$.

Lemma 4 Let $\overline{\mathcal{C}}$ be any clustering for an instance $G_{\mathcal{I}}$ of $S D G$ containing only basic coalitions of the five types $t_{0}, \ldots, t_{4}$. Starting from $\overline{\mathcal{C}}$, the game admits a Nash equilibrium with at least the same social welfare.

Proof. In order to prove that the game admits a Nash equilibrium starting from any $\overline{\mathcal{C}}$, we can define a suitable potential function which strictly increases each time an agent makes an improving move. 
Therefore, we argue that the game has the finite improvement path property, and thus it admits a Nash equilibrium.

Given any clustering $\overline{\mathcal{C}}$ including only coalitions of the types $t_{0}, \ldots, t_{4}$, and denoted by $\alpha_{i}$ the number of coalitions of type $t_{i}$ included in $\overline{\mathcal{C}}$, we consider the following potential function, coincident with the social welfare:

$$
\phi(\overline{\mathcal{C}})=S W(\overline{\mathcal{C}})=\sum_{i=0}^{4} \alpha_{i} S W\left(t_{i}\right) .
$$

First of all we can notice that agents in $K_{i} \cup\left\{x_{i}\right\}$ are stable in every coalition in $\overline{\mathcal{C}}$ :

- Any agent $z \in K_{i}$ would be disconnected in any other coalition $t_{j} \cup\{z\}$, and thus by moving her utility would decrease to zero.

- Any agent $x_{i}$ would obtain a utility zero by moving from $t_{i}$ to $t_{j}$ whenever the new formed coalition $t_{j} \cup\left\{x_{i}\right\}$ is disconnected. This happens for instance when $x_{i}$ moves from $t_{i}$, for $i \in\{3,4\}$, or when $x_{i}$ moves to $t_{j}=t_{0}$. On the other hand, for $i \in\{0,1,2\}$, agent $x_{i}$ has utility $u_{x_{i}}\left(t_{i}\right)=\frac{3+i+\frac{1}{2}}{5+i} \geq \frac{7}{10}$, and she would decrease her utility by moving to any other basic coalition $t_{j}$ with $j \geq 1$ even if $t_{j} \cup\left\{x_{i}\right\}$ is connected. In fact:

$$
\begin{aligned}
& -u_{x_{i}}\left(t_{1} \cup\left\{x_{i}\right\}\right)=\frac{1+\frac{1}{2}+\frac{3}{3}+\frac{1}{4}}{7}=\frac{11}{28}<\frac{7}{10} \leq u_{x_{i}}\left(t_{i}\right) ; \\
& -u_{x_{i}}\left(t_{j} \cup\left\{x_{i}\right\}\right) \leq \frac{2+\frac{1}{2}+\frac{3+(j-2)}{3}+\frac{1}{4}}{7+(j-1)} \leq \frac{15}{32}<\frac{7}{10} \leq u_{x_{i}}\left(t_{i}\right), \text { for } j \in\{2,3\} ; \\
& \text { - } u_{x_{i}}\left(t_{4} \cup\left\{x_{i}\right\}\right) \leq \frac{2+\frac{1}{2}+\frac{1}{3}}{5}=\frac{17}{30}<\frac{7}{10} \leq u_{x_{i}}\left(t_{i}\right) .
\end{aligned}
$$

It remains to examine agents $y \in Y$ included in any $t_{i}$. In every basic coalition we can compute the following utilities $u_{y}\left(t_{i}\right)$ :

$$
\begin{aligned}
& u_{y}\left(t_{i}\right)=\frac{1+\frac{3+(i-1)}{2}+\frac{1}{3}}{6+(i-1)}= \begin{cases}\frac{17}{36} \approx 0.472 & \text { if } i=1 \\
\frac{10}{21} \approx 0.476 & \text { if } i=2 \\
\frac{23}{48} \approx 0.479 & \text { if } i=3\end{cases} \\
& u_{y}\left(t_{4}\right)=\frac{1+\frac{2}{2}}{4}=\frac{1}{2}=0.5 .
\end{aligned}
$$

Since no agent $y$ can move to $t_{3}$ or $t_{4}$ without decreasing her utility to zero, we can focus only on possible moves from $t_{i}$ to $t_{j}$ with $j \leq 2$. Moreover, since if $y$ moves from $t_{i}$ to $t_{j}$ we either obtain $t_{j+1}$ or a coalition where $y$ is disconnected, by Equation (8) it is evident that $t_{3}$ and $t_{4}$ are already stable, because every $y$ in $t_{3}$ and $t_{4}$ would not increase her utility by moving to $t_{j}$ with $j \leq 2$, and that every $y \in t_{i}$ for $i \in\{1,2\}$ can improve her utility only by moving to a coalition of type $t_{j}$ for $i \leq j \leq 2$.

Therefore, in a clustering $\overline{\mathcal{C}}$ all the coalitions of type $t_{0}, t_{3}$ and $t_{4}$ are already Nash stable, whereas if $\overline{\mathcal{C}}$ is not Nash stable it necessarily depends on the unstability of some agent $y$ in $t_{1}$ or $t_{2}$. In the following we prove that when such an agent $y$ performs an improving move to $t_{j}$, for $i \leq j \leq 2$, the social welfare strictly increases, and the new coalitions are still of types $t_{0}, \ldots, t_{4}$.

For the sake of simplicity, let us denote by $\left(\overline{\mathcal{C}}, t_{i}, t_{j}\right)$ the improving deviation of an agent $y$ from a cluster of type $t_{i}$ to a cluster of type $t_{j}$, in $\overline{\mathcal{C}}$. Since we know that $y$ can move only from $t_{i}$ to $t_{j}$, for $i \in\{1,2\}$ and $i \leq j \leq 2$, we can distinguish three cases: 
1. if $t_{i}=t_{j}=t_{1}$, i.e., $y$ moves from a coalition of type $t_{1}$ to another coalition of type $t_{1}$, from two coalitions of type $t_{1}$ we obtain two coalitions respectively of type $t_{0}$ (stable) and $t_{2}$, and therefore: $\phi\left(\left(\overline{\mathcal{C}}, t_{1}, t_{1}\right)\right)-\phi(\overline{\mathcal{C}})=S W\left(t_{0}\right)+S W\left(t_{2}\right)-2 S W\left(t_{1}\right)=\frac{19}{5}+\frac{94}{21}-2 \cdot \frac{37}{9}=$ $\frac{869}{105}-\frac{74}{9} \approx 8.27-8.22=0.05 ;$

2. if $t_{i}=t_{j}=t_{2}$, i.e., $y$ moves from a coalition of type $t_{2}$ to another coalition of type $t_{2}$, from two coalitions of type $t_{2}$ we obtain two coalitions of type $t_{1}$ and $t_{3}$ (stable), and therefore: $\phi\left(\left(\overline{\mathcal{C}}, t_{2}, t_{2}\right)\right)-\phi(\overline{\mathcal{C}})=S W\left(t_{1}\right)+S W\left(t_{3}\right)-2 S W\left(t_{2}\right)=\frac{37}{9}+\frac{39}{8}-2 \cdot \frac{94}{21}=\frac{647}{72}-\frac{188}{21} \approx$ $8.98-8.95=0.03$

3. if $t_{i}=t_{1}, t_{j}=t_{2}$ i.e., $y$ moves from a coalition of type $t_{1}$ to a coalition of type $t_{2}$, from two coalitions of type $t_{1}$ and $t_{2}$ we obtain two coalitions of type $t_{0}$ and $t_{3}$ (both stable), and therefore: $\phi\left(\left(\overline{\mathcal{C}}, t_{1}, t_{2}\right)\right)-\phi(\overline{\mathcal{C}})=S W\left(t_{0}\right)+S W\left(t_{3}\right)-\left(S W\left(t_{1}\right)+S W\left(t_{2}\right)\right)=\frac{19}{5}+\frac{39}{8}-$ $\left(\frac{37}{9}+\frac{94}{21}\right)=\frac{347}{40}-\frac{541}{63} \approx 8.675-8.587=0.088$.

In every case the social welfare strictly increases. Since the number of possible clusterings is finite, this implies that starting from any clustering $\overline{\mathcal{C}}$ consisting of coalitions of type $t_{0}, \ldots, t_{4}$, there exists a finite improvement path converging to a Nash stable clustering (still containing coalitions of type $t_{0}, \ldots, t_{4}$ ) with a strictly higher social welfare.

We can now prove the following corollary.

Corollary 1 Let $\mathcal{C}$ be any Nash stable clustering for an instance $G_{\mathcal{I}}$ of SDG including only basic coalitions. Every $x_{i}$-coalition $C=K_{i} \cup\left\{x_{i}\right\} \cup Y_{i}$ of type $t_{3}$ can be split in two sub-coalitions $C^{\prime}=K_{i}$ and $C^{\prime \prime}=\left\{x_{i}\right\} \cup Y_{i}$, obtaining a new stable clustering $\mathcal{C}^{\prime}=\mathcal{C} \backslash\{C\} \cup\left\{C^{\prime}, C^{\prime \prime}\right\}$ with a strictly higher social welfare.

Proof. Consider the clustering $\mathcal{C}^{\prime}$ obtained from $\mathcal{C}$ by splitting a coalition $C$ of type $t_{3}$ (see Figure 9) in two sub-coalitions $C^{\prime}=K_{i}$ and $C^{\prime \prime}=\left\{x_{i}\right\} \cup Y_{i}$ corresponding to type $t_{4}$. The stability of $\mathcal{C}^{\prime}$ directly arises from the proofs of Lemmas 2 and 4 . Moreover, we can easily verify that $\mathcal{C}^{\prime}$ has a strictly higher social welfare. In fact:

$$
\begin{aligned}
S W\left(t_{3}\right) & =\frac{\left(3+\frac{1}{2}+\frac{3}{3}\right)+3\left(4+\frac{3}{2}\right)+\left(6+\frac{1}{2}\right)+3\left(1+\frac{5}{2}+\frac{1}{3}\right)}{8}=\frac{\frac{9}{2}+\frac{33}{2}+\frac{13}{2}+\frac{23}{2}}{8} \\
& =\frac{39}{8}=4.875
\end{aligned}
$$

and therefore, by Equations (6) and (9), $S W\left(t_{4}\right)-S W\left(t_{3}\right)=\frac{21}{4}-\frac{39}{8}=\frac{3}{8}=0.375$.

On the basis of these considerations, we can state the following result.

Corollary 2 Any best Nash equilibrium for an instance $G_{\mathcal{I}}$ of $S D G$ must include only basic coalitions of the 4 types $t_{0}, t_{1}, t_{2}, t_{4}$.

We are now ready to claim the following main theorem, whose proof comes directly from the following Lemmas 5 and 6 below.

Theorem 2 Computing a best Nash equilibrium for SDGs is NP-hard. 
Lemma 5 If there is an exact cover for the input instance $\mathcal{I}$ of $R X C 3$, then there exists a Nash equilibrium for the instance $G_{\mathcal{I}}$ of $S D G$ with social welfare at least $\frac{257}{20} p$.

Proof. If there is an exact cover, then there is a clustering consisting of exactly $p$ coalitions of type $t_{4}$ associated to the triples of the cover, and $(m-p)=2 p$ remaining coalitions of type $t_{0}$. By Lemma 4 such a clustering is stable and the claim follows by simply summing up the utilities of all the agents, i.e., $\frac{21}{4} p+\frac{19}{5}(2 p)$.

Lemma 6 If there is not an exact cover for the input instance $\mathcal{I}$ of $R X C 3$, then every Nash equilibrium for the instance $G_{\mathcal{I}}$ of $S D G$ has social welfare strictly less than $\frac{257}{20} p$.

Proof. Assume then that there is not an exact cover. By Corollary 2, the stable clusterings achieving the best social welfare include only basic coalitions of the 4 types $t_{0}, t_{1}, t_{2}, t_{4}$. Consider any such stable clustering $\mathcal{C}$. For each $i \in[m]$, we rearrange the social welfare of each coalition of $\mathcal{C}$ in such a way that $\frac{19}{5}$ is always accounted to the nodes $z_{i, 1}, z_{i, 2}, z_{i, 3}, z_{i, 4}, x_{i}$, and the surplus is equally shared among the agents $y \in Y_{i}$ belonging to the coalition. We have three cases for charging such $y$ agents, depending on how many of them are contained in each coalition: in case of $t_{1}$ there is a single $y$ agent and we account to her all the remaining social welfare, that is $\frac{14}{45} \approx 0.31$; in the case $t_{2}$ there are two $y$ agents, and we account $\frac{71}{210} \approx 0.338$ to each of them; in the case $t_{4}$ there are three $y$ agents, and we account to each $\frac{29}{60} \approx 0.483$. Notice that, since $\frac{29}{60}>\frac{71}{210}>\frac{14}{45}$, a clustering with social welfare equal to $\frac{29}{60} m+\frac{19}{5} m=\frac{257}{60} m=\frac{257}{20} p$ can be obtained if and only if $\frac{29}{60}$ is accounted to all the $y$ nodes, which would imply the existence of an exact cover: a contradiction.

\section{Price of Anarchy and Price of Stability}

In this section we first provide matching upper and lower bounds on the price of anarchy of SDGs. We then focus on the price of stability, and prove that there exists an instance of SDGs for which the lower bound on the $\operatorname{PoS}$ is $\frac{6}{5}-\epsilon$, for any $\epsilon>0$. Finally, we study the PoS for graphs with girth 4 and girth at least 5 , the girth being the length of the shortest cycle in the graph. In the sequel we call star a tree consisting of one vertex (the center) adjacent to all the other vertices (the leaves).

Theorem 3 There exists a class of SDGs having price of anarchy $\Theta(n)$.

Proof. We recall that, by the remark made in Section 2, we can focus on SDGs for non-singleton and connected graphs. By the definition of the game, the social welfare of any clustering is upper bounded by $n-1$ (and therefore OPT $\leq n-1$ ), as every agent has utility at most $\frac{n-1}{n}$. Such an upper bound can be attained only by the grand coalition in complete connected graphs. On the other hand, since in any equilibrium $\mathcal{C}$ every agent has at least one neighbor in her coalition, thus having utility at least $\frac{1}{n}$, it is $S W(\mathcal{C}) \geq 1$.

It remains to show that there exists a class of SDGs having price of anarchy $\Omega(n)$. To this end, for any positive integer $n^{\prime}$, consider the graph of $n=8 n^{\prime}$ nodes depicted in Figure 11. In the Nash stable solution $\mathcal{C}$ illustrated in Figure 12,

$$
S W(\mathcal{C})=\frac{n}{4} \cdot \frac{2\left(1+\frac{1}{2}+\frac{1}{3}\right)+2\left(2+\frac{1}{2}\right)}{4}=\frac{n}{4} \cdot \frac{26}{4}=\frac{13}{3} n^{\prime} .
$$


Another Nash stable solution $\mathcal{C}^{\prime}$ is shown in Figure 13. In this case, the social welfare is

$$
S W\left(\mathcal{C}^{\prime}\right)=2 \cdot \frac{\left[2\left(1+\frac{1}{2}+\frac{1}{3}\right)+2\left(2+\frac{1}{2}\right)\right] \cdot \frac{\frac{n}{2}}{4}}{n / 2}=2 \cdot \frac{\frac{26}{3} n^{\prime}}{4 n^{\prime}}=\frac{13}{3}
$$

and therefore

$$
\mathrm{PoA} \geq \frac{\frac{13 n^{\prime}}{3}}{\frac{13}{3}}=n^{\prime}=\Omega(n)
$$

Thus, the claim follows.

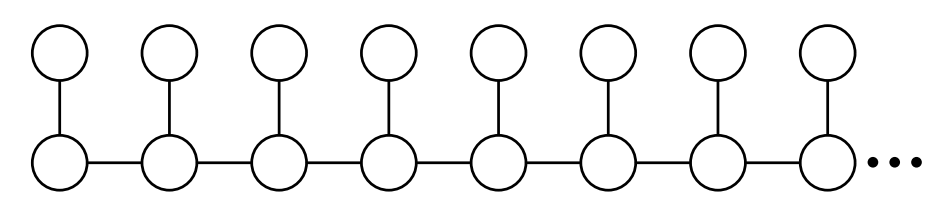

Figure 11: A SDG with $n$ agents having $\mathrm{PoA}=\Omega(n)$.
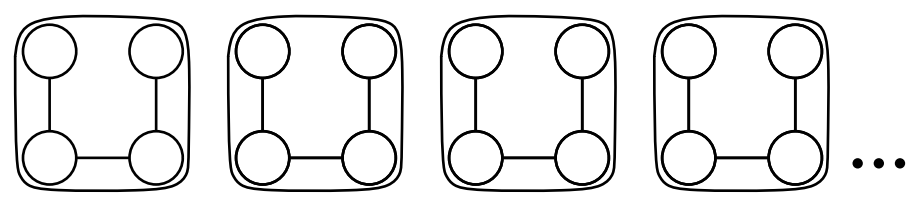

Figure 12: A Nash stable solution with $S W=\frac{13 n}{24}$ for the SDG depicted in Figure 11.

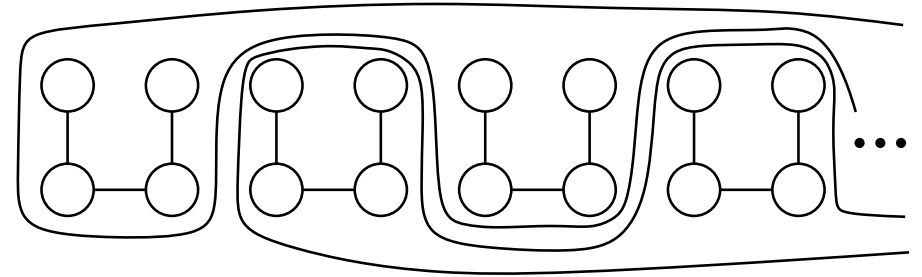

Figure 13: A Nash stable solution with $S W=\frac{13}{3}$ for the SDG depicted in Figure 11.

Let us now turn our attention to the PoS. A first general lower bound is established in the following theorem.

Theorem 4 There exists a class of SDGs having PoS at least $\frac{6}{5}-\epsilon$, for any arbitrarily small $\epsilon>0$.

Proof. For any integer $\nu \geq 3$, consider a graph of $2 \nu+2$ nodes consisting of a clique of $\nu+1$ agents $K_{\nu+1}=\left\{z_{i} \mid i \in[\nu+1]\right\}$, each connected to the center $x$ of a star of $\nu$ leaves $Y=\left\{y_{i} \mid i \in[\nu]\right\}$. An example of the graph for $\nu=3$ is depicted in Figure 14(a).

Let $S=\{x\} \cup Y$ be the set of the agents in the star. Consider the clustering $\mathcal{C}=\left\{K_{\nu+1}, S\right\}$ (see Figure 14(b)), with social welfare

$$
S W(\mathcal{C})=\nu+\frac{\nu}{(\nu+1)} \frac{(\nu+3)}{2}=\frac{\nu(3 \nu+5)}{2(\nu+1)} .
$$




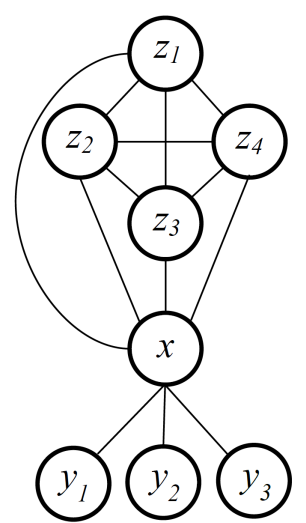

(a)

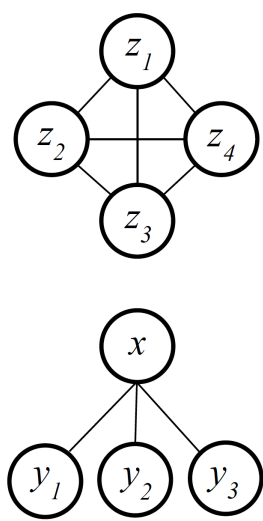

(b)

Figure 14: A SDG (a) and a corresponding clustering which is not Nash stable (b).

$\mathcal{C}$ is not Nash stable, since agent $x$ can increase her utility from $\frac{\nu}{\nu+1}$ to $\frac{\nu+1}{\nu+2}$ by moving to $K_{\nu+1}$.

We now prove that the grand coalition (Figure 14(a)) is the only (and consequently, the best) Nash stable solution.

First of all, in any stable clustering, each agent $y_{i} \in Y$ cannot be isolated, and thus must be connected to $x$, otherwise she would have utility 0 . Therefore, all agents in $S$ must be in the same coalition. Moreover, there can be no pair of coalitions containing only agents of $K_{\nu+1}$, otherwise all agents in one coalition of minimum cardinality would move one after the other to the other coalition to increase their utility (even starting from two subsets of $K_{\nu+1}$ of the same size). As a consequence, any stable clustering cannot contain more than two coalitions, as being all the agents of $S$ in a same coalition, the remaining two coalitions should be contained in $K_{\nu+1}$. Thus, in order to prove that also all agents in $K_{\nu+1}$ must be in the same coalition of $x$, it remains to show that any clustering consisting of two different coalitions $C_{1} \subset K_{\nu+1}$ and $C_{2}=V \backslash C_{1}$ (i.e., $C_{2}$ includes $S$ and the remaining agents of $K_{\nu+1}$ ) is not stable. Arguing by contradiction, assume that there exists such a stable clustering $\mathcal{C}=\left\{C_{1}, C_{2}\right\}$. Let $\nu_{1}=\left|C_{1}\right|>1\left(C_{1}\right.$ cannot be singleton $)$ and $\nu_{2}=\left|K_{\nu+1} \cap C_{2}\right| \geq 1$ (as mentioned above, if $\nu_{2}=0$ the clustering is not stable) be the number of agents of $K_{\nu+1}$ belonging to $C_{1}$ and $C_{2}$ respectively, with $\nu_{1}+\nu_{2}=\nu+1$.

Let $z_{1}$ be an agent in $C_{1}$ and $z_{2}$ be an agent in $K_{\nu+1} \cap C_{2}$. The utilities of $z_{1}$ and $z_{2}$ are the following:

- $u_{z_{1}}\left(C_{1}\right)=\frac{\nu_{1}-1}{\nu_{1}}$ : if $z_{1}$ moves, her utility becomes $u_{z_{1}}\left(C_{2} \cup\left\{z_{1}\right\}\right)=\frac{\nu_{2}+\frac{\nu}{2}+1}{\nu+\nu_{2}+2}$;

- $u_{z_{2}}\left(C_{2}\right)=\frac{\nu_{2}+\frac{\nu}{2}}{\nu+\nu_{2}+1}$ : if $z_{2}$ moves, her utility becomes $u_{z_{2}}\left(C_{1} \cup\left\{z_{2}\right\}\right)=\frac{\nu_{1}}{\nu_{1}+1}$.

We want to prove that (i) if agent $z_{1}$ does not want to move, then $z_{2}$ prefers to move, and (ii) if agent $z_{2}$ does not want to move, then $z_{1}$ prefers to move.

It is easy to see that, for any choice of $\nu_{1}$ and $\nu_{2}$ :

- $u_{z_{1}}\left(C_{1}\right)<u_{z_{2}}\left(C_{1} \cup\left\{z_{2}\right\}\right)$, and

- $u_{z_{2}}\left(C_{2}\right)<u_{z_{1}}\left(C_{2} \cup\left\{z_{1}\right\}\right)$. 
Now, $z_{1}$ does not want to move when $u_{z_{1}}\left(C_{1}\right) \geq u_{z_{1}}\left(C_{2} \cup\left\{z_{1}\right\}\right)$. This implies that $u_{z_{2}}\left(C_{2}\right)<$ $u_{z_{1}}\left(C_{2} \cup\left\{z_{1}\right\}\right) \leq u_{z_{1}}\left(C_{1}\right)<u_{z_{2}}\left(C_{1} \cup\left\{z_{2}\right\}\right)$, that is $z_{2}$ wants to move. Similarly, when $z_{2}$ does not want to move, that is when $u_{z_{2}}\left(C_{2}\right) \geq u_{z_{2}}\left(C_{1} \cup\left\{z_{2}\right\}\right)$, we obtain $u_{z_{1}}\left(C_{1}\right)<u_{z_{2}}\left(C_{1} \cup\left\{z_{2}\right\}\right) \leq$ $u_{z_{2}}\left(C_{2}\right)<u_{z_{1}}\left(C_{2} \cup\left\{z_{1}\right\}\right)$, that is $z_{1}$ wants to move. In both cases we can conclude that the clustering would not be stable. Therefore, the grand coalition is the only Nash stable solution. Its social welfare is $S W(V)=(\nu+1) \cdot u_{z_{i}}(V)+u_{x}(V)+\nu \cdot u_{y_{i}}(V)=\frac{\nu(5 \nu+11)+4}{4 \nu+4}$, where $u_{z_{i}}(V)=\frac{\nu+1+\frac{\nu}{2}}{2 \nu+2}$ for all $i \in[\nu+1], u_{x}(V)=\frac{2 \nu+1}{2 \nu+2}$ and $u_{y_{i}}(V)=\frac{1+\nu}{2 \nu+2}$ for all $i \in[\nu]$.

Hence, the ratio $\frac{S W(\mathcal{C})}{S W(V)}=\frac{2 \nu(3 \nu+5)}{\nu(5 \nu+11)+4}$ tends to $\frac{6}{5}$ as $\nu$ tends to infinity, proving the theorem.

The following lower bound on the PoS for graphs of girth 4 holds.

Theorem 5 There exists an instance of SDGs, in which the underlying graph has girth 4, that has $\mathrm{PoS}$ at least $169 / 160=1.05625$.

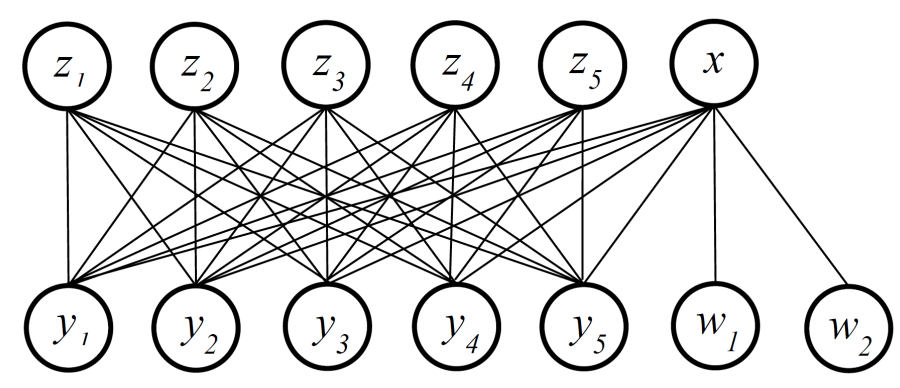

Figure 15: A SDG with $\mathrm{PoS} \geq \frac{169}{160}$.

Proof. Consider the graph in Figure 15 and let us denote $Z=\left\{z_{1}, \ldots, z_{5}\right\}, Y=\left\{y_{1}, \ldots, y_{5}\right\}$ and $S=\left\{x, w_{1}, w_{2}\right\}$. We first show that there exists an unstable clustering that achieves high social welfare, and then that any stable solution achieves quite less.

More precisely, consider the clustering $\overline{\mathcal{C}}=\{Y \cup Z, S\}$. Since $u_{z_{i}}(\overline{\mathcal{C}})=u_{y_{i}}(\overline{\mathcal{C}})=\frac{7}{10}$ for all $i \in[5], u_{x}(\overline{\mathcal{C}})=\frac{2}{3}$ and $u_{w_{1}}(\overline{\mathcal{C}})=u_{w_{2}}(\overline{\mathcal{C}})=\frac{1}{2}$, it has social welfare $S W(\overline{\mathcal{C}})=10 \cdot \frac{7}{10}+\frac{2}{3}+1=\frac{26}{3}$, but it is not stable, as $x$ can move and increase her utility from $u_{x}(S)=\frac{2}{3}$ to $u_{x}(Y \cup Z \cup\{x\})=$ $\frac{5+\frac{5}{2}}{11}=\frac{15}{22}$ by switching coalition.

We now prove that the best stable solution is the grand coalition, that has $S W(V)=5 u_{z_{i}}(V)+$ $5 u_{y_{i}}(V)+u_{x}(V)+2 u_{w_{i}}(V)=\frac{320}{39}$, where $u_{z_{i}}(V)=\frac{5+\frac{5}{2}+\frac{2}{3}}{13}=\frac{49}{78}$ and $u_{y_{i}}(V)=\frac{6+\frac{6}{2}}{13}=\frac{9}{13}$ for $i \in[5], u_{x}(V)=\frac{7+\frac{5}{2}}{13}=\frac{19}{26}$ and $u_{w_{i}}=\frac{1+\frac{6}{2}+\frac{5}{3}}{13}=\frac{17}{39}$ for $i \in\{1,2\}$. The result then derives by the ratio between the above two social welfares.

Notice first that, in any stable clustering, $w_{1}$ and $w_{2}$ must be in the same coalition of $x$, otherwise they would have utility 0 and could improve their utility by moving.

Let $\mathcal{C}$ be a stable clustering not being the grand coalition. In the remainder of the proof we will make use of the following properties of $\mathcal{C}$ :

- connection-property: every coalition of $\mathcal{C}$ containing an agent $z \in Z$ (resp. $y \in Y$ ) must contain at least one agent $y \in Y$ (resp. $z \in Z \cup\{x\}$ ). In fact, otherwise $z$ (resp. $y$ ) would have utility 0 and could improve her utility by moving. 
- half-property: an agent $z \in Z$ (resp. $y \in Y$ ) has utility strictly greater than $1 / 2$ if and only if it is in a coalition with at least two agents of $Y$ (resp. of $Z \cup\{x\}$ ). In fact, recalling the definition of the utilities of SDGs, if $z \in Z$ (resp. $y \in Y$ ) is connected with one agent of $Y$ (resp. $Z \cup\{x\}$ ) (by the connection-property at least one must be in her coalition), she would have only one agent at distance 1 and eventual other ones at distance at least 2 , so her utility would be at most $1 / 2$. On the other hand, if $z$ (resp. $y$ ) is connected with two agents of $Y$ (resp. of $Z \cup\{x\}$ ), then she has at least 2 nodes at distance 1, eventual nodes at distance 2 and at most 2 nodes at distance 3 , so her utility would be strictly greater than $1 / 2$.

Having in mind the above properties, we distinguish two cases depending on whether $\mathcal{C}$ does not contain or contains a coalition $C$ with at least two agents of $Z \cup\{x\}$.

In the former case, by the connection-property and the fact that agents $w_{1}$ and $w_{2}$ must always be with $x$, up to symmetries the only possibility for $\mathcal{C}$ is that $\mathcal{C}=\left\{\left\{z_{1}, y_{1}\right\}, \ldots,\left\{z_{5}, y_{5}\right\}, S\right\}$. Such a clustering has social welfare $S W(\mathcal{C})=5+\frac{5}{3}=\frac{20}{3}$, which is strictly less than the $\frac{320}{39}$ one obtained by the grand coalition.

So let us assume then that $\mathcal{C}$ contains a coalition $C$ with at least two agents of $Z \cup\{x\}$. In the remaining part of the proof we show that under this assumption $\mathcal{C}$ cannot be stable.

Notice first that in $\mathcal{C}$ there cannot be any coalition $C_{1}$ with only one agent in $Z \cup\{x\}$. In fact, by the connection-property, $C_{1}$ should have at least one agent $y \in Y$, that by the half-property would be interested in moving to $C$. This also implies that $\mathcal{C}$ can contain at most 3 coalitions, so let us assume there is also a coalition $C_{2}$ with at least two agents of $Y$. Therefore, in a similar way, there cannot be a coalition containing a single agent $y \in Y$ and at least one agent $z \in Z$, because by the half-property $z$ would be interested in moving to $C_{2}$.

As a consequence of the above observations, every coalition not containing $x$ must have at least two agents of $Z$ and at least two agents of $Y$.

If $\mathcal{C}$ has two coalitions $C_{1}$ and $C_{2}$ not containing $x$, then recalling that $\mathcal{C}$ has at most 3 coalitions and that by the connection-property the remaining coalition $C_{3}$ containing $x$ either has both an agent of $Z$ and $Y$ or none of them, up to symmetries the following cases can hold:

i. $C_{1}=\left\{z_{1}, z_{2}, y_{1}, y_{2}\right\}, C_{2}=\left\{z_{3}, z_{4}, y_{3}, y_{4}\right\}, C_{3}=\left\{z_{5}, y_{5}, x, w_{1}, w_{2}\right\}$,

ii. $C_{1}=\left\{z_{1}, z_{2}, z_{3}, y_{1}, y_{2}\right\}, C_{2}=\left\{z_{4}, z_{5}, y_{3}, y_{4}, y_{5}\right\}, C_{3}=\left\{x, w_{1}, w_{2}\right\}$,

iii. $C_{1}=\left\{z_{1}, z_{2}, y_{1}, y_{2}\right\}, C_{2}=\left\{z_{3}, z_{4}, z_{5}, y_{3}, y_{4}, y_{5}\right\}, C_{3}=\left\{x, w_{1}, w_{2}\right\}$.

In case i. by the half-property $z_{5}$ is interested to move to $C_{1}$ or $C_{2}$. In case ii. $z_{3}$ can improve her utility from $u_{z_{3}}\left(C_{1}\right)=\frac{1+1+\frac{1}{2}+\frac{1}{2}}{5}=\frac{3}{5}$ to $u_{z_{3}}\left(C_{2} \cup\left\{z_{3}\right\}\right)=\frac{1+1+1+\frac{1}{2}+\frac{1}{2}}{6}=\frac{2}{3}>\frac{3}{5}$. Finally in case iii. $z_{2}$ can improve her utility from $u_{z_{2}}\left(C_{1}\right)=\frac{1+1+\frac{1}{2}}{4}=\frac{5}{8}$ to $u_{z_{2}}\left(C_{2} \cup\left\{z_{2}\right\}\right)=\frac{1+1+1+\frac{1}{2}+\frac{1}{2}+\frac{1}{2}}{7}=$ $\frac{9}{14}>\frac{5}{8}$.

Therefore, since $\mathcal{C}$ cannot have two coalitions $C_{1}$ and $C_{2}$ not containing $x$, the only possibility left is that $\mathcal{C}$ consists of just two coalitions (recall that we are under the assumption that $\mathcal{C}$ is not the grand coalition).

By exploiting symmetries and the above constraints, we can describe all possible clusterings containing exactly two coalitions using just two parameters, $\alpha$ and $\beta$. All such clusterings (up to symmetries) are:

$$
\left\{\left\{\left\{z_{1}, \ldots, z_{\alpha}, y_{1}, \ldots, y_{\beta}\right\},\left\{z_{\alpha+1}, \ldots, z_{5}, y_{\beta+1}, \ldots, y_{5}, x, w_{1}, w_{2}\right\}\right\} \mid 2 \leq \alpha \leq 4,2 \leq \beta \leq 3\right\} .
$$


By means of a case analysis we show that they are all unstable, as there is always at least one agent that has an improving move.

Let $C_{1}=\left\{z_{1}, \ldots, z_{\alpha}, y_{1}, \ldots, y_{\beta}\right\}$ and $C_{2}=\left\{z_{\alpha+1}, \ldots, z_{5}, y_{\beta+1}, \ldots, y_{5}, x, w_{1}, w_{2}\right\}$. Then:

- If $\alpha=2$ agent $y_{1}$ moves. In fact, her original utility is $u_{y_{1}}\left(C_{1}\right)=\frac{2+\frac{\beta-1}{2}}{2+\beta}$, while her new one is $u_{y_{1}}\left(C_{2} \cup\left\{y_{1}\right\}\right)=\frac{4+\frac{7-\beta}{2}}{12-\beta}$, and for any $\beta$ in our domain, $u_{y_{1}}\left(C_{1}\right)<u_{y_{1}}\left(C_{2} \cup\left\{y_{1}\right\}\right)$.

- If $\beta=3$ agent $z_{5}$ moves. In fact, her original utility is $u_{z_{5}}\left(C_{2}\right)=\frac{2+\frac{5-\alpha}{2}+\frac{2}{3}}{10-\alpha}$, while her new one is $u_{z_{5}}\left(C_{1} \cup\left\{z_{5}\right\}\right)=\frac{3+\frac{\alpha}{2}}{4+\alpha}$, and $u_{z_{5}}\left(C_{2}\right)<u_{z_{5}}\left(C_{1} \cup\left\{z_{5}\right\}\right)$ for any $\alpha$ such that $3 \leq \alpha \leq 4$.

- The remaining case is $3 \leq \alpha \leq 4$ and $\beta=2$. Under this assumption $y_{5}$ moves. In fact, her original utility is $\frac{8-\alpha}{11-\alpha}$, while her new one is $\frac{\alpha+1}{\alpha+3}$, which is strictly greater for any $\alpha$ such that $3 \leq \alpha \leq 4$

In conclusion, there cannot neither be any stable clustering consisting of two coalitions, hence the claim.

Finding an upper bound on the price of stability in SDGs seems to be a challenging task. However, we can make progress in this direction by considering more restrictive settings. More precisely, we provide an upper bound of $\frac{5}{4}$ on the price of stability on graphs with girth at least 5 . Notice that this result is not comparable with the lower bound on PoS obtained in Theorem 4, since the lower bound is given by a construction having girth less than 5 . Before proving such a lower bound, we also show that if we have the promise that the graph has girth 4 , then there exists an instance of SDGs in which the only stable solution is the grand coalition.

Lemma 7 There exists an instance of SDGs in which the underlying graph has girth 4 and the only stable solution is the grand coalition.

Proof. Consider the graph in Figure 16 and let us analyze all possible ways for partitioning the nodes in a stable way.

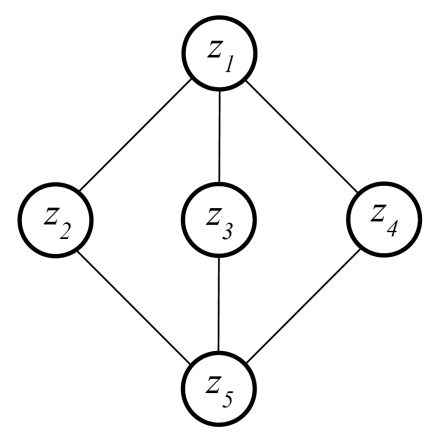

Figure 16: A graph with girth 4.

First of all, notice that an agent cannot be isolated and that, if a coalition is not connected, an agent will move because her utility would be zero. So, besides the grand coalition, we can only partition $V$ in two connected coalitions of size 2 and 3 . We only consider the case $\mathcal{C}=$ 
$\left\{\left\{z_{1}, z_{2}\right\},\left\{z_{3}, z_{4}, z_{5}\right\}\right\}$, as all the others are symmetric. $\mathcal{C}$ is not stable, since $z_{1}$ can increase her utility from $\frac{1}{2}$ to $\frac{5}{8}$. This implies the theorem.

For a greater girth the following result holds.

Lemma 8 Given any graph with girth greater than 4 , it is possible to obtain a stable solution $\mathcal{C}$ with $S W(\mathcal{C}) \geq \frac{n}{2}$ by partitioning the graph into stars.

Proof. The following simple procedure allows to determine a partition of the graph into stars, each of at least 2 nodes, together with the corresponding stable clustering. Once constructed a rooted spanning tree $T$ of the graph $G$, let $x$ be one of the deepest leaves and $y$ be her parent in $T$. Put $y$ and all her children ( $x$ included) in the same coalition and iterate the procedure on the residual tree, until either the tree becomes empty or only the root is left. In this last case insert the root in the last formed coalition. Notice that every coalition induces exactly a star subgraph, as such a subgraph has all the leaves connected to a center and, by the assumption on the girth, there can not be edges between leaves.

It is easy to check that in a star all the leaves have utility $\frac{1}{2}$, while the center has utility at least $\frac{1}{2}$. For what concerns the stability, consider any agent in a star. Since the girth is greater than 4 , she can have only one neighbor in a different star, hence she cannot achieve utility greater than $\frac{1}{2}$ by moving, hence the claim.

Hence, we can finally state the following upper bound on the PoS for graphs of girth greater than 4 .

Theorem 6 The upper bound on the PoS of SDGs in which the underlying graph has girth strictly greater than 4 is $\frac{1}{2}+\frac{1}{\sqrt{2}} \approx 1.207$.

Proof. Consider any clustering $\mathcal{C}$ and let $C_{1}, \ldots, C_{k}$ be the non-singleton coalitions in $\mathcal{C}$, that is such that $\left|C_{i}\right| \geq 2$ for $i \in[k]$.

Let $n_{i}=\left|C_{i}\right|, E_{i}$ be the subset of the edges induced by $C_{i}$, and $\delta_{x}$ be the degree of agent $x$ in the subgraph induced by her coalition. We can give an upper bound to the utility of $x$ by considering her neighbors in her coalition $C_{i}$ at distance 1 and all the other agents in $C_{i}$ as if they were at distance 2. Hence, the contribute $S W_{i}$ of any coalition $C_{i}$ to the social welfare for $i \in[k]$, is

$$
\begin{aligned}
S W_{i} & \leq \sum_{x \in C_{i}} \frac{\delta_{x}+\frac{n_{i}-\delta_{x}-1}{2}}{n_{i}}=\sum_{x \in C_{i}} \frac{\frac{n_{i}-1}{2}+\frac{\delta_{x}}{2}}{n_{i}}= \\
& =\frac{n_{i}-1}{2}+\sum_{x \in C_{i}} \frac{\delta_{x}}{2 n_{i}}=\frac{n_{i}-1}{2}+\frac{2\left|E_{i}\right|}{2 n_{i}}= \\
& =\frac{n_{i}-1}{2}+\frac{\left|E_{i}\right|}{n_{i}} .
\end{aligned}
$$

Since the subgraph induced by $C_{i}$ cannot have a girth lower than the one of $G$, and thus has girth greater than 4, from Dutton and Brigham (1991) we know that $\left|E_{i}\right| \leq \frac{n_{i} \sqrt{n_{i}-1}}{2}$. Thus,

$$
S W_{i} \leq \frac{n_{i}-1}{2}+\frac{\sqrt{n_{i}-1}}{2}
$$


and

$$
\begin{aligned}
S W(\mathcal{C})= & \sum_{i \in[k]} S W_{i} \leq \frac{\sum_{i \in[k]}\left(n_{i}-1\right)+\sum_{i \in[k]} \sqrt{n_{i}-1}}{2}= \\
& =\frac{n-k+\sum_{i \in[k]} \sqrt{n_{i}-1}}{2} \leq \frac{n-k+k \sqrt{\frac{n}{k}-1}}{2} .
\end{aligned}
$$

From Lemma 8, we can guarantee a social welfare of at least $\frac{n}{2}$, hence

$$
\operatorname{PoS} \leq \frac{\frac{n-k+k \sqrt{\frac{n}{k}-1}}{2}}{\frac{n}{2}} \leq \frac{1}{2}+\frac{1}{\sqrt{2}},
$$

where the last inequality comes from standard maximization arguments, thus proving the theorem.

\section{Conclusions}

We investigated Nash stability in SDGs.Several issues remain open. First of all, we have shown that there is a class of SDGs for which a $\frac{6}{5}$ lower bound on the price of stability holds; it would be nice to provide a corresponding upper bound. Another relevant question that naturally emerges is whether there exists a polynomial time algorithm for determining the existence of a Nash stable clustering for SDGs different from the grand coalition. As we have seen, this is not guaranteed for graphs with girth less or equal to 4 . We notice that, besides this computational issue, a deeper understanding of the conditions for the existence of such non trivial equilibria would be particularly important for providing better bounds on the price of stability. A related open question is that of identifying special graphs in which a best equilibrium or a best non stable clustering can be computed in polynomial time. Finally, it would be interesting to generalize our results to weighted graphs and to consider classes of hedonic and fractional hedonic games induced by other classical centrality measures, like the ones presented by Gomez et al. (2003). On this respect, it would be particularly worth to consider models in which being a singleton is not the worst choice.

\section{Acknowledgements}

A preliminary version of this work has appeared in the proceedings of the 31st Conference on Artificial Intelligence (AAAI), February 4-9, 2017, San Francisco, California, USA (Balliu et al., 2017a). We thank Giovanna Varricchio and Bojana Kodric for fruitful discussions about the content of this paper. Many thanks to the anonymous reviewers for their helpful comments and suggestions.

This work was partially supported by the Academy of Finland, Grant 285721, and by the Italian MIUR PRIN 2017 Project ALGADIMAR “Algorithms, Games, and Digital Markets”.

\section{References}

Anshelevich, E., Dasgupta, A., Kleinberg, J., Tardos, E., Wexler, T., \& Roughgarden, T. (2004). The price of stability for network design with fair cost allocation. In Proceedings of the 45th Annual IEEE Symposium on Foundations of Computer Science, FOCS '04, pp. 295-304, Washington, DC, USA. IEEE Computer Society. 
Apt, K., \& Witzel, A. (2009). A generic approach to coalition formation. International Game Theory Review, 11(03), 347-367.

Aziz, H., Brandt, F., \& Harrenstein, P. (2014). Fractional hedonic games. In Proceedings of the 2014 International Conference on Autonomous Agents and Multi-agent Systems, AAMAS '14, pp. 5-12, Richland, SC. International Foundation for Autonomous Agents and Multiagent Systems.

Aziz, H., Brandt, F., \& Seedig, H. (2011). Stable partitions in additively separable hedonic games. In The 10th International Conference on Autonomous Agents and Multiagent Systems - Volume 1, AAMAS '11, pp. 183-190. International Foundation for Autonomous Agents and Multiagent Systems.

Aziz, H., Brandt, F., \& Seedig, H. (2013). Computing desirable partitions in additively separable hedonic games. Artificial Intelligence, 195, 316-334.

Aziz, H., Gaspers, S., Gudmundsson, J., Mestre, J., \& Täubig, H. (2015). Welfare maximization in fractional hedonic games. In Proceedings of the Twenty-Fourth International Joint Conference on Artificial Intelligence, IJCAI 2015, Buenos Aires, Argentina, July 25-31, 2015, pp. 461-467.

Aziz, H., \& Savani, R. (2016). Hedonic Games, chap. 15. Cambridge University Press.

Aziz, H., Brandt, F., \& Harrenstein, P. (2013). Pareto optimality in coalition formation. Games and Economic Behavior, 82, 562-581.

Ballester, C. (2004). NP-completeness in hedonic games. Games and Economic Behavior, 49(1), $1-30$.

Balliu, A., Flammini, M., Melideo, G., \& Olivetti, D. (2017a). Nash stability in social distance games. In Proceedings of the 31st AAAI Conference on Artificial Intelligence, February 4-9, 2017, AAAI '17, pp. 342-348. AAAI Press.

Balliu, A., Flammini, M., \& Olivetti, D. (2017b). On Pareto optimality in social distance games. In Proceedings of the 31st AAAI Conference on Artificial Intelligence, February 4-9, 2017, AAAI '17, pp. 349-355. AAAI Press.

Banerjee, S., Konishi, H., \& Sönmez, T. (2001). Core in a simple coalition formation game. Social Choice and Welfare, 18(1), 135-153.

Bilò, V., Fanelli, A., Flammini, M., Monaco, G., \& Moscardelli, L. (2014). Nash stability in fractional hedonic games. In Web and Internet Economics - 10th International Conference, WINE 2014, Beijing, China, December 14-17, 2014. Proceedings, pp. 486-491.

Bilò, V., Fanelli, A., Flammini, M., Monaco, G., \& Moscardelli, L. (2015). On the price of stability of fractional hedonic games. In Proceedings of the 2015 International Conference on Autonomous Agents and Multiagent Systems, AAMAS 2015, Istanbul, Turkey, May 4-8, 2015, pp. 1239-1247.

Bloch, F., \& Diamantoudi, E. (2011). Noncooperative formation of coalitions in hedonic games. International Journal of Game Theory, 40(2), 263-280.

Bogomolnaia, A., \& Jackson, M. (2002). The stability of hedonic coalition structures. Games and Economic Behavior, 38(2), 201-230. 
Boldi, P., \& Vigna, S. (2014). Axioms for centrality. Internet Mathematics, 10(3-4), 222-262.

Brandl, F., Brandt, F., \& Strobel, M. (2015). Fractional hedonic games: Individual and group stability. In Proceedings of the 2015 International Conference on Autonomous Agents and Multiagent Systems, AAMAS '15, pp. 1219-1227. International Foundation for Autonomous Agents and Multiagent Systems.

Brânzei, S., \& Larson, K. (2011). Social distance games. In IJCAI 2011, Proceedings of the 22nd International Joint Conference on Artificial Intelligence, Barcelona, Catalonia, Spain, July 16-22, 2011, pp. 91-96.

Cechlárová, K., \& Romero-Medina, A. (2001). Stability in coalition formation games. International Journal of Game Theory, 29(4), 487494.

Dreze, J., \& Greenberg, J. (1980). Hedonic coalitions: Optimality and stability. Econometrica, 48(4), 987-1003.

Dutton, R. D., \& Brigham, R. (1991). Edges in graphs with large girth. Graphs and Combinatorics, 7(4), 315-321.

Elkind, E., Fanelli, A., \& Flammini, M. (2016). Price of Pareto optimality in hedonic games. In AAAI, pp. 475-481. AAAI Press.

Elkind, E., \& Wooldridge, M. (2009). Hedonic coalition nets. In Proceedings of The 8th International Conference on Autonomous Agents and Multiagent Systems - Volume 1, AAMAS '09, pp. 417-424, Richland, SC. International Foundation for Autonomous Agents and Multiagent Systems.

Feldman, M., Lewin-Eytan, L., \& Naor, J. (2015). Hedonic clustering games. ACM Trans. Parallel Comput., 2(1), 4:1-4:48.

Flammini, M., Monaco, G., Moscardelli, L., Shalom, M., \& Zaks, S. (2018). Online coalition structure generation in graph games. In Proceedings of the 17th International Conference on Autonomous Agents and MultiAgent Systems, AAMAS 2018, Stockholm, Sweden, July 10-15, 2018, pp. 1353-1361.

Flammini, M., Monaco, G., \& Zhang, Q. (2017). Strategyproof mechanisms for additively separable hedonic games and fractional hedonic games. In Approximation and Online Algorithms - 15th International Workshop, WAOA 2017, Vienna, Austria, September 7-8, 2017, Revised Selected Papers, pp. 301-316.

Gairing, M., \& Savani, R. (2010). Computing stable outcomes in hedonic games. In Algorithmic Game Theory - Third International Symposium, SAGT 2010, Athens, Greece, October 18-20, 2010. Proceedings, pp. 174-185.

Gomez, D., Gonzlez-Arangena, E., Manuel, C., Owen, G., del Pozo, M., \& Tejada, J. (2003). Centrality and power in social networks: a game theoretic approach. Mathematical Social Sciences, 46(1), 27 - 54 .

Gonzalez, T. (1985). Clustering to minimize the maximum intercluster distance. Theoretical Computer Science, 38, 293-306.

Kaklamanis, C., Kanellopoulos, P., \& Patouchas, D. (2018). On the price of stability of social distance games. In Deng, X. (Ed.), Algorithmic Game Theory, pp. 125-136, Cham. Springer International Publishing. 
Kaklamanis, C., Kanellopoulos, P., \& Papaioannou, K. (2016). The price of stability of simple symmetric fractional hedonic games. In Algorithmic Game Theory - 9th International Symposium, SAGT 2016, Liverpool, UK, September 19-21, 2016. Proceedings, pp. 220-232.

Koutsoupias, E., \& Papadimitriou, C. (1999). Worst-case equilibria. In Proceedings of the 16th Annual Symposium On Theoretical Aspects of Computer science, pp. 404-413.

McPherson, M., Lovin, L., \& Cook, J. (2001). Birds of a feather: Homophily in social networks. Annual Review of Sociology, 27(1), 415-444.

Monaco, G., Moscardelli, L., \& Velaj, Y. (2018). Stable outcomes in modified fractional hedonic games. In Proceedings of the 17th International Conference on Autonomous Agents and MultiAgent Systems, AAMAS 2018, Stockholm, Sweden, July 10-15, 2018, pp. 937-945.

Olsen, M. (2009). Nash stability in additively separable hedonic games and community structures. Theory of Computing Systems, 45(4), 917-925.

Olsen, M. (2012). On defining and computing communities. In Eighteenth Computing: The Australasian Theory Symposium, CATS 2012, Melbourne, Australia, January 2012, pp. 97-102.

Papadimitriou, C. (2001). Algorithms, games, and the internet. In Automata, Languages and Programming, 28th International Colloquium, ICALP 2001, Crete, Greece, July 8-12, 2001, Proceedings, pp. 1-3. 